\title{
Fat jet signature of a heavy neutrino at a lepton collider
}

\author{
Sabyasachi Chakraborty, ${ }^{1,2, *}$ Manimala Mitra, ${ }^{3,4, \uparrow}$ and Sujay Shil ${ }^{3,4, *}$ \\ ${ }^{1}$ Department of Physics, Florida State University, Tallahassee, Florida 32306, USA \\ ${ }^{2}$ Department of Theoretical Physics, Tata Institute of Fundamental Research, Mumbai 400005, India \\ ${ }^{3}$ Homi Bhabha National Institute, Training School Complex, Anushakti Nagar, Mumbai 400085, India \\ ${ }^{4}$ Institute of Physics, Sachivalaya Marg, Bhubaneswar, Odisha 751005, India
}

(Received 11 November 2018; published 10 July 2019)

\begin{abstract}
We explore the discovery prospect of a very heavy neutrino at the proposed $e^{+} e^{-}$collider for two different c.m. energies $\sqrt{s}=1.4$ and $3 \mathrm{TeV}$. We consider production of a heavy neutrino via $s$ - and $t$-channel processes and its subsequent prompt decays leading to semileptonic final states, along with significant missing momentum. For our choice of masses, the gauge boson produced from heavy neutrino decay is highly boosted, leading to a fat jet. We carry out a detailed signal and background analysis for $e^{ \pm}+j_{\text {fat }}+p$ (missing four-momentum) final state using both cut-based and multivariate techniques. We show that a heavy neutrino of mass $600-2700 \mathrm{GeV}$ and active-sterile mixing $\left|V_{e N}\right|^{2} \sim 10^{-5}$ can be probed with $5 \sigma$ significance at an $e^{+} e^{-}$collider after collecting $\mathcal{L}=500 \mathrm{fb}^{-1}$ of data. We find that the sensitivity reach at an $e^{+} e^{-}$collider is an order of magnitude enhanced compared to the LHC.
\end{abstract}

DOI: $10.1103 /$ PhysRevD.100.015012

\section{INTRODUCTION}

The experimental observation of neutrino oscillations in different oscillation experiments has conclusively given evidence that neutrinos have tiny $\mathrm{eV}$ masses and nonzero mixings [1]. This is a definitive indication for the existence of beyond the Standard Model physics (BSM physics). The solar and atmospheric mass square differences from neutrino oscillation experiments are about $\Delta m_{12}^{2} \sim 10^{-5} \mathrm{eV}^{2}$ and $\left|\Delta m_{13}^{2}\right| \sim 10^{-3} \mathrm{eV}^{2}$, and the mixing angles are $\theta_{12} \sim 33^{\circ}, \theta_{23} \sim 42^{\circ}$, and $\theta_{13} \sim 8^{\circ}$. Augmented with stringent limits from Planck, the sum of light neutrino masses is bounded from above at $\Sigma_{i} m_{i} \leq 0.12-0.66 \mathrm{eV}$ [2], where the range corresponds to the different datasets considered. A number of BSM extensions have been proposed to explain small neutrino masses. A few of them are the seesaw paradigm $[3,4]$, neutrino mass generation through radiative processes [5-8], R-parity violating supersymmetry [9], etc.

Among the above, one of the most appealing frameworks of light neutrino mass generation is seesaw, where Majorana masses of the light neutrinos are generated from

\footnotetext{
*sabya@hep.fsu.edu

†manimala@iopb.res.in

"sujay@iopb.res.in
}

Published by the American Physical Society under the terms of the Creative Commons Attribution 4.0 International license. Further distribution of this work must maintain attribution to the author(s) and the published article's title, journal citation, and DOI. Funded by SCOAP ${ }^{3}$. lepton number violating dimension-5 operators $L L H H / \Lambda$ $[3,4]$. There can be a few different variations of seesaw: type I [10-16], type II [17-20], and type III [21]. In type-I and type-III seesaw, heavy neutral leptons are included in the model. Furthermore, in type III, the neutral lepton is a part of the $S U(2)_{L}$ triplet fermionic field. In type-II seesaw, an $S U(2)_{L}$ triplet Higgs with hypercharge $Y=+2$ is included. Both type I and type II can be embedded in a left-right symmetric model [22-24] with an extended gauge group. The other very popular seesaw scenario is the inverse seesaw [25-27], where the smallness of the light neutrino mass is protected by an enhanced lepton number symmetry of the Lagrangian.

Most of the UV completed seesaw models contain the Standard Model (SM) gauge singlet heavy neutrino $N$. Depending on the mass of the gauge singlet neutrinos and their mixings with the active neutrino states, seesaw can be tested at colliders [28-47], as well as in other noncollider experiments, such as neutrinoless double beta decay [48-54]; lepton flavor violating processes $\ell_{i} \rightarrow \ell_{j} \gamma$, $\mu \rightarrow 3 e, \mu \rightarrow e$ conversion in nuclei [55,56]; rare-meson decays [57-59], etc. Among the collider studies, LHC searches mostly focus on the charged-current production mode, i.e., heavy neutrino production in $p p \rightarrow \ell^{ \pm} N$, followed by the subsequent decays of $N$. The smoking gun signature that confirms the Majorana nature of $N$ corresponds to the same-sign dilepton + dijet final state $[60,61]$. However, the golden trilepton channel [62] associated with missing energy is very promising, owing to the smaller background rate. The active-sterile mixing $V_{l N}$ has been constrained in the range $\left|V_{l N}\right|^{2}<10^{-5}$ for the mass of 
the heavy neutrino $10<M_{N}<50 \mathrm{GeV}$ [63]. For higher masses, in particular, for the TeV range $M_{N}$, the LHC cross section becomes significantly smaller. Hence, the bound on the active-sterile mixing relaxes considerably. Other than the LHC searches, heavy neutrinos can also be looked into using an $e^{+} e^{-}$collider, as well as an $e^{-} p$ collider [64-66]. See [67-77] for previous discussions of the heavy neutrino searches at an $e^{+} e^{-}$collider. Most of these works discuss the prospect of observation at an $e^{+} e^{-}$collider for $M_{N} \lesssim 500 \mathrm{GeV}$. For lower masses, $M_{N} \lesssim 500 \mathrm{GeV}$, the ILC can probe active-sterile mixing $\left|V_{e N}\right|^{2} \sim 10^{-4}$, with $\mathcal{L}=$ $100 \mathrm{fb}^{-1}$ of data. There is a moderate to ultraheavy mass range $M_{N} \sim \mathrm{TeV}$ or beyond that can further be explored in the proposed $e^{+} e^{-}$collider Compact Linear Collider (CLIC) [78-81], in its higher c.m. energy run with $\sqrt{s}=1.4$ and $3 \mathrm{TeV}$. Note, that $M_{N}$ up to $1 \mathrm{TeV}$ can also be probed at the ILC, in its $1 \mathrm{TeV}$ run. We stress that the model signature for a very heavy $N$ is quite distinct from that of $M_{N}$ in the $100 \mathrm{GeV}$ mass range, which we explore in detail. See [80,82-93] for the discovery prospect of different BSM scenarios at the CLIC.

In this work, we study the discovery prospect of a heavy neutrino in the intermediate to very high mass range at an $e^{+} e^{-}$collider. We consider two different c.m. energies $\sqrt{s}=$ 1.4 and $3 \mathrm{TeV}$, respectively, that are relevant for the CLIC. Contrary to the LHC, the production cross section of a superheavy neutrino at an $e^{+} e^{-}$collider is fairly large. We consider two different mass ranges: $M_{N}=600-1200 \mathrm{GeV}$, which can be probed at the $1.4 \mathrm{TeV}$ run of the CLIC, and $M_{N}=1300-2700 \mathrm{GeV}$, which can be discovered with $3 \mathrm{TeV}$ c.m. energy. We consider the production mode $e^{+} e^{-} \rightarrow \nu_{e} N$ and the subsequent decays of $N$ into an electron $e^{ \pm}$and $W^{\mp}$ gauge boson. We further consider the hadronic decay modes of $W^{ \pm}$. For such a heavy $N$, the $W^{ \pm}$'s are highly boosted. Hence, the quarks from $W^{ \pm}$are collimated, leading to a single fat jet. Therefore, the final state is $e^{ \pm}+j_{\text {fat }}+p$. We pursue an in-depth study for this final state, with both cutbased and multivariate analysis (MVA). We show that a heavy neutrino with mass 600-2700 GeV and mixing $\left|V_{e N}\right|^{2} \sim$ $10^{-5}$ can be discovered with $5 \sigma$ significance at an $e^{+} e^{-}$ collider with $\mathcal{L} \sim 500 \mathrm{fb}^{-1}$ luminosity, which is an order of magnitude improvement as opposed to the LHC limit.

The paper is organized as follows. In Sec. II, we discuss the interactions of the heavy neutrino with SM particles. In Sec. II A, we discuss our model signature. Followed by this, in Sec. III A, we present a detailed event analysis using cut-based techniques for the signal and background. In Sec. III B, we optimize our search strategies using MVA, which further enhances the signal sensitivity. The results of both the cut-based and MVA analysis are discussed in Sec. III C. Finally, we present our conclusions in Sec. IV.

\section{INTERACTIONS OF HEAVY NEUTRINO}

The heavy neutrino, as discussed in the introduction, can be a part of different seesaw models, such as type-I and
type-III seesaw, inverse seesaw, etc. For our discussion, we follow a model independent framework, with the assumption that the heavy neutrinos are SM gauge singlet states and, hence, do not directly interact with SM particles. Any interaction of the heavy neutrino with the SM gauge bosons and Higgs is therefore governed by its mixing with the active neutrinos. Note that, for inverse seesaw, a large active-sterile mixing $\left(\left|V_{e N}\right|^{2} \simeq 10^{-5}\right)$ that we consider in this work is naturally in agreement with the constraint from light neutrino mass measurement. In other scenarios, such as type-I or type-III seesaw, accidental cancellation in the light neutrino mass matrix will be required to justify such a large mixing [48].

We consider $n$-generation right-handed (RH) neutrinos $N_{R_{\beta}}^{\prime}$ (in the flavor basis) that mix with the SM light neutrinos $\nu_{L_{\alpha}}$. The light neutrinos in their flavor basis can be expressed in terms of the fields in the mass basis $\left(\nu_{m_{i}}, N_{R_{j}}^{c}\right)$ as follows:

$$
\nu_{L_{\alpha}}=U \nu_{L_{i}}+V N_{R_{j}}^{c} .
$$

In the equation above, $\nu_{L_{i}}$ refers to the active neutrinos in their mass basis and $N_{R_{j}}^{c}$ is the conjugate field of RH neutrino $N_{R}$, written in the mass basis. The matrix $U$ is the Pontecorvo-Maki-Nakagawa-Sakata (PMNS) matrix, and $V$ parametrizes the mixing of the active neutrinos with the gauge singlet heavy states. Owing to the active-sterile mixing $V$, the heavy neutrinos $N_{j}$ in their mass basis interact with the SM particles, through the charged-current, neutral-current interactions [30,70]:

$$
-\mathcal{L}_{C C}=\frac{g}{\sqrt{2}} W_{\mu}^{-} \bar{\ell}_{i} \gamma^{\mu} P_{L} V_{i j} N_{j}+\text { H.c. },
$$

and

$-\mathcal{L}_{N C}=\frac{g}{2 \cos \theta_{w}} Z_{\mu}\left\{\left(U^{\dagger} V\right)_{i j} \bar{\nu}_{i} \gamma^{\mu} P_{L} N_{j}+\right.$ H.c. $\}$.

The interaction of the heavy neutrinos with SM Higgs has the following form:

$$
-\mathcal{L}_{H}=\frac{g M_{j}}{4 M_{W}} H\left\{\left(U^{\dagger} V\right)_{i j} \bar{\nu}_{i} P_{R} N_{j}+\text { H.c. }\right\} .
$$

In the above $M_{j}$ represents the mass of the heavy neutrino $N_{j}$. We consider a diagonal basis for the charged leptons, and hence no further mixing from charged lepton sector enters in Eq. (2.2). The partial decay widths of different decay modes have the following expression:

$$
\Gamma\left(N \rightarrow \ell^{-} W^{+}\right)=\frac{g^{2}}{64 \pi}\left|V_{\ell N}\right|^{2} \frac{M_{N}^{3}}{M_{W}^{2}}\left(1-\frac{M_{W}^{2}}{M_{N}^{2}}\right)^{2}\left(1+2 \frac{M_{W}^{2}}{M_{N}^{2}}\right),
$$



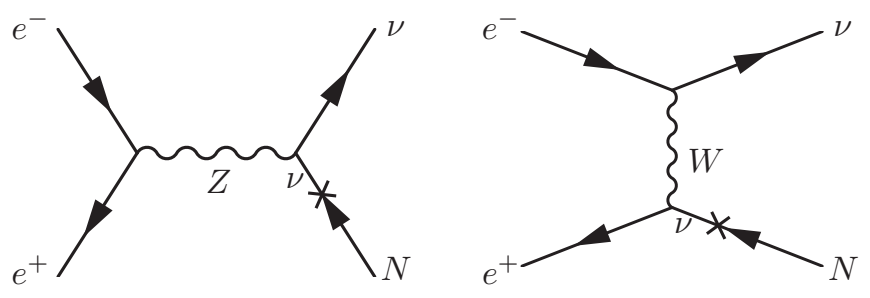

FIG. 1. Feynman diagrams for heavy neutrino production at a lepton collider. For our analysis, we consider both the $\nu_{e}$ and $\bar{\nu}_{e}$ states.

$$
\Gamma\left(N \rightarrow \nu_{\ell} Z\right)=\frac{g^{2}}{128 \pi}\left|V_{\ell N}\right|^{2} \frac{M_{N}^{3}}{M_{W}^{2}}\left(1-\frac{M_{Z}^{2}}{M_{N}^{2}}\right)^{2}\left(1+2 \frac{M_{Z}^{2}}{M_{N}^{2}}\right),
$$

$\Gamma\left(N \rightarrow \nu_{\ell} H\right)=\frac{g^{2}}{128 \pi}\left|V_{\ell N}\right|^{2} \frac{M_{N}^{3}}{M_{W}^{2}}\left(1-\frac{M_{H}^{2}}{M_{N}^{2}}\right)^{2}$.

For the heavy neutrino significantly more massive than the SM gauge bosons and Higgs, i.e., $M_{N} \gg M_{W}, M_{Z}, M_{H}$, the branching ratio is approximated as $\operatorname{Br}\left(N \rightarrow \ell^{ \pm} W^{\mp}\right)$ : $\operatorname{Br}\left(N \rightarrow \nu_{\ell} Z\right): \operatorname{Br}\left(N \rightarrow \nu_{\ell} H\right)=2: 1: 1$. We show the variation of the branching ratio with the mass of $N$ in Fig. 2 . For $M_{N} \geq 600 \mathrm{GeV}$, which is of our interest, the leading branching $\operatorname{Br}(N \rightarrow \ell W) \sim 50 \%$. This has a significant impact on our choice of final states, as will be clear from the next section.

Note that, in addition to the collider experiments, heavy neutrinos can also be probed through other experimental signals, such as neutrinoless double beta decay $(0 \nu 2 \beta)$. However, this relies on the assumption that the heavy neutrinos are of a Majorana nature. For a pure type-I seesaw scenario, we estimate the constraint on active-sterile mixing that can originate from $0 \nu 2 \beta$. In particular, we consider the lower limit on half-life of the $0 \nu 2 \beta$ process $T_{0 \nu}^{1 / 2}>8.0 \times 10^{25} \mathrm{yr}$ at $90 \%$ C.L., given by GERDA [94].
The half-life $T_{1 / 2}^{0 \nu}$, active-sterile mixing $V_{e N}$, and the mass of the heavy neutrino $M_{N}$ are related as follows:

$$
\frac{1}{T_{0 \nu}^{1 / 2}} \simeq G_{0 \nu} \mathcal{M}_{N}^{2}\left|\frac{V_{e N}^{2} m_{p}}{M_{N}}\right|^{2}
$$

In the above, $G_{0 \nu}$ is the phase space factor, $G_{0 \nu}=$ $5.77 \times 10^{-15} \mathrm{yr}^{-1}[95]\left(G_{0 \nu}=G_{0 \nu}^{(0)} g_{A}^{4}\right) ; \mathcal{M}_{N}$ is the nuclear matrix element for ${ }^{76} \mathrm{Ge}$; and $m_{p}$ is the mass of the proton. As an example, we consider $\mathcal{M}=232.8$ [96]. Using the above equation, we find that for heavy neutrino mass $M_{N}=(1.0-2.7) \mathrm{TeV}$, active-sterile mixing $\left|V_{e N}\right|^{2} \simeq$ $\left(0.63 \times 10^{-5}-1.70 \times 10^{-5}\right)$. In the $\mathrm{TeV}$ mass range, constraints from $0 \nu 2 \beta$ and a future $e^{+} e^{-}$collider are comparable (for present and future collider constraints, see Fig. 13). For pseudo-Dirac heavy neutrinos with a tiny mass splitting, as in the case with inverse seesaw, their contribution in $0 \nu 2 \beta$-decay tends to cancel each other. Hence, the constraint on active-sterile mixing will be significantly relaxed. On the other hand, a signal such as $\ell+j_{\text {fat }}+\not p$, which we analyze in this work, is present irrespective of the nature of heavy neutrinos.

\section{A. Production and decay at a lepton collider}

The heavy neutrino interacts with the charged leptons and SM gauge bosons. Due to the interaction of the heavy neutrinos $N_{j}$ with $l^{ \pm}-W^{\mp}$ and $\nu_{\ell}-Z, N_{j}$ can be produced at a lepton collider. The Feynman diagram for the production process $e^{+} e^{-} \rightarrow \nu_{e} N$ is shown in Fig. 1, and the cross section is given in the left panel of Fig. 2, for c.m. energies $\sqrt{s}=1.4$ and $3 \mathrm{TeV}$. For comparison, we also show the production cross section at LHC, with $13 \mathrm{TeV}$ c.m. energy for both the channels $p p \rightarrow e^{ \pm} N$ and $p p \rightarrow \nu_{e} N$. For the hundred $\mathrm{GeV}-\mathrm{TeV}$ mass range $200<M_{N}<2900 \mathrm{GeV}$, the normalized cross section at a lepton collider varies from $\sigma \sim\left(10^{2}-6.7\right) \mathrm{pb}$, which is larger than the production cross section at the LHC by
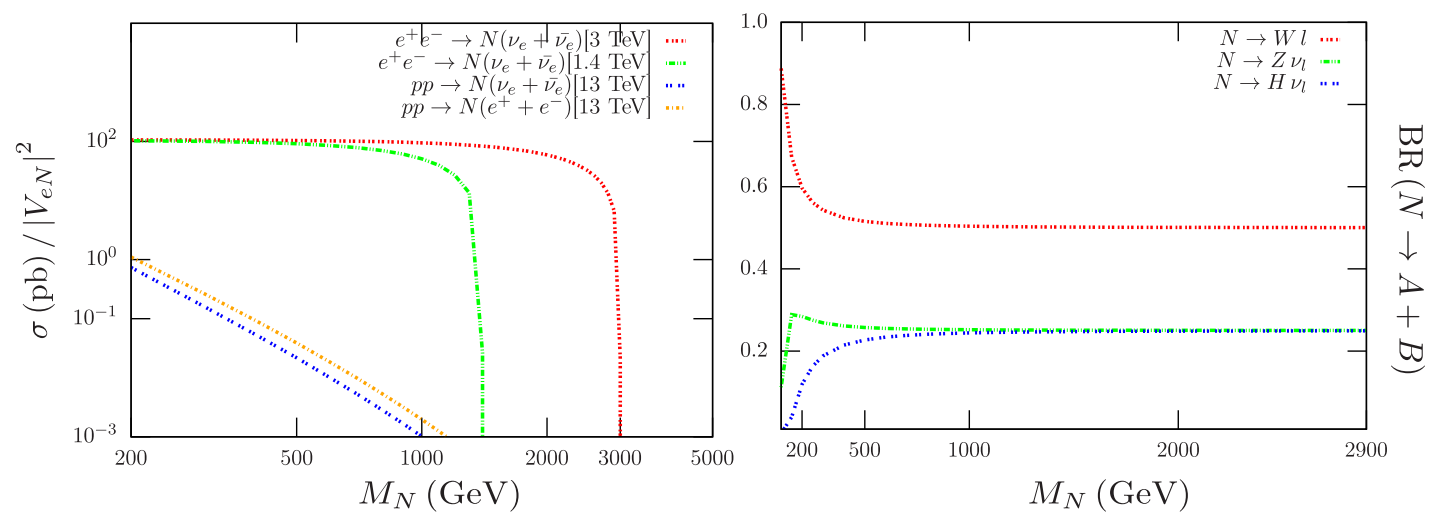

FIG. 2. Left panel: We plot the production cross section of the heavy neutrino normalized by the active-sterile mixing parameter. We also compare our results for the compact linear collider with the LHC. Right panel: Variation of branching ratio of $N$ vs mass. The different decay modes are $N \rightarrow \nu_{e} Z, N \rightarrow l^{ \pm} W^{\mp}$, and $N \rightarrow \nu_{e} H$ states. 
at least $\mathcal{O}\left(10^{2}\right)$. To probe heavier $M_{N}$ at LHC, a relatively large partonic c.m. energy is required. The fall in the cross section for higher $M_{N}$ occurs due to the drop of the parton distribution function. Furthermore, the channel $p p \rightarrow \nu_{\ell} N$ suffers additional suppression as compared to $e^{+} e^{-} \rightarrow \nu_{\ell} N$, due to smaller electromagnetic coupling.

The channel $e^{+} e^{-} \rightarrow \nu_{e} N$ has also been explored before in [70] for lower c.m. energies $\sqrt{s}=250$ and $500 \mathrm{GeV}$. It has been inferred that a mixing down to $\left|V_{e N}\right|^{2} \sim 10^{-4}$ can be probed at a linear collider up to $M_{N}=400 \mathrm{GeV}$ with $100 \mathrm{fb}^{-1}$ of data. Recently, $13 \mathrm{TeV}$ LHC searches looked for the conventional dilepton + dijet signature [61], but also for the golden channel trilepton associated with missing energy $p p \rightarrow \ell N \rightarrow \ell^{ \pm} \ell^{\mp} \ell^{ \pm}+E_{T}$ [63]. While for relatively lower mass $10<M_{N}<50 \mathrm{GeV}$, the bound on the active-sterile mixing is $\left|V_{e N}\right|^{2} \lesssim 10^{-5}$ [63], and for $M_{N} \sim 100 \mathrm{GeV}$, this is about $10^{-2}$, for the medium mass range $M_{N} \gtrsim 500 \mathrm{GeV}$, the constraint is significantly relaxed. Almost no constraint from collider searches appears for $M_{N}$ in the $\mathrm{TeV}$ range. The cross section at a lepton collider, on the other hand, is large even for a heavier neutrino mass, that is within the kinematic threshold. Hence, the heavy neutrino of mass several hundred $\mathrm{GeV}$ or $\mathrm{TeV}$ should have a higher discovery prospect at a lepton collider. For the analysis that we pursue in this work, we focus on the moderate to high mass regime of the heavy neutrino, starting from $600 \mathrm{GeV}$, up to around $3 \mathrm{TeV}$.

Subsequent decay of the heavy neutrino produces a number of final states that can be probed in the lepton collider:

(i) $e^{+} e^{-} \rightarrow \nu_{\ell} N \rightarrow \nu_{\ell} \ell^{ \pm} W^{\mp} \rightarrow \ell j j+\not p$,

(ii) $e^{+} e^{-} \rightarrow \nu_{\ell} N \rightarrow \nu \nu Z \rightarrow j j+\not p, \ell^{+} \ell^{-}+\not p$,

(iii) $e^{+} e^{-} \rightarrow \nu_{\ell} N \rightarrow \nu \nu H \rightarrow b \bar{b}+\not p, \tau^{+} \tau^{-}+\not p$.

For the very high mass regime of the heavy neutrino, the produced gauge bosons will be boosted. Hence, the jets from the gauge boson decay would be collimated, leading to a fat jet. We consider the channel with the highest branching ratio of $N$, i.e., $N \rightarrow \ell^{ \pm} W^{\mp}$ (with $\ell=e^{+}, e^{-}$), and hadronic decays of the $W^{ \pm}$. Therefore, our model signature is

(i) $e^{+} e^{-} \rightarrow \nu_{e} N \rightarrow e^{ \pm} W^{\mp} \nu_{e} \rightarrow e^{ \pm}+\not p+j_{\text {fat }}$.

In our analysis, we include both the production modes $e^{+} e^{-} \rightarrow \nu_{e} N$ and $e^{+} e^{-} \rightarrow \bar{\nu}_{e} N$. For simplicity, in the above we consider only one decay channel of the heavy neutrino $N \rightarrow e^{ \pm} W^{\mp}$. This occurs if the active-sterile mixing $V \simeq I$ is nearly diagonal. However, for a nondiagonal mixing matrix, $N$ can decay to all three flavors $e, \mu, \tau$. The $\tau$ will again decay either hadronically or leptonically. Therefore, in the more generic scenario with all the flavors, the final state leptons would be $e^{ \pm}$and $\mu^{ \pm}$.

\section{COLLIDER ANALYSIS}

We perform both the cut-based and multivariate analysis to probe heavy neutrinos at collider. To simulate the signal events, we write the interactions of the heavy neutrinos [Eqs. (2.2)-(2.4)] in FeynRules [97,98]. The generated Universal FeynRules Output (UFO) [99] model files are then fed into Monte Carlo (MC) event generator MadGraph5 aMC@NLO [100] to generate event samples for the analysis. The partonic events are then passed through Pythia8 [101] for showering and hadronization, and detector simulation has been carried out with Delphes3.4.1 [102], with the card corresponding to the detector for international linear collider. We use the CambridgeAchen jet clustering algorithm [103] to form jets, where we consider the radius parameter $R=1.0$. For the signal, we consider the active-sterile mixing $\left|V_{e N}\right|=0.01$, so that heavy neutrino $N$ has large decay width $\left(\Gamma_{N}=\right.$ $2.77 \times 10^{-2} \mathrm{GeV}-2.58 \mathrm{GeV}$ for $M_{N}=600-2700 \mathrm{GeV}$ ), and the decay of $N$ occurs within the detector. We generate background as $e^{ \pm}+\nu_{e} / \bar{\nu}_{e}+j j$ in MadGraph5 aMC@NLO and follow the same set of tools for analysis. The background $e^{ \pm} \nu_{e} / \bar{\nu}_{e} j j$ arises from $W^{ \pm} W^{\mp}$, but also from other production processes ( $t$-channel mediated diagrams, off-shell gauge boson contributions, etc). In our analysis, we omit the $\tau \nu_{\tau} j j$ background, as after taking into account the leptonic branching ratios, the cross section becomes an order of magnitude smaller $(\sigma \sim 12 \mathrm{fb})$. Moreover, the electron that originates from $\tau$ decay largely fails to pass our selection criterion.

We split the analysis in two different categories: (a) a heavy neutrino with mass $600-1200 \mathrm{GeV}$ can be probed with $\sqrt{s}=1.4 \mathrm{TeV}$ c.m. energy and (b) a more massive heavy neutrino up to mass $M_{N} \sim 3.0 \mathrm{TeV}$ can be probed with $\sqrt{s}=3 \mathrm{TeV}$. We reiterate that the final state that we demand has a single isolated charged lepton $e^{ \pm}$, one fat jet $j_{\text {fat }}$ with jet radius $R=1.0$, and missing four-momentum $\not p$.

\section{A. Cut-based analysis}

\section{1. $M_{N}=600-1200 \mathrm{GeV}$ with $\sqrt{s}=1.4 \mathrm{TeV}$}

At the c.m. energy $\sqrt{s}=1.4 \mathrm{TeV}$, a heavy neutrino mass up to $M_{N} \sim 1400 \mathrm{GeV}$ can be explored kinematically. As can be seen from Fig. 2, the fall in the cross section occurs near the kinematic threshold. However, a wide range of masses starting from a few hundred $\mathrm{GeV}$ up to $\mathrm{TeV}$ has a fairly large production cross section. As an illustrative example, we consider $M_{N}=900 \mathrm{GeV}$. For this choice of mass, the production cross section is $\sigma\left(e^{+} e^{-} \rightarrow \nu_{e} N\right)=$ $17.8 \mathrm{pb}$, for the active-sterile mixing $\left|V_{e N}\right|=I$. A production cross section proportional to $\left|V_{e N}\right|^{2}$ falls down to $\sigma\left(e^{+} e^{-} \rightarrow \nu_{e} N\right)=1.78 \mathrm{fb}$ for mixing $\left|V_{e N}\right|=10^{-2}$. In the subsequent analysis, we consider the above-mentioned value of the active-sterile mixing, which is in agreement with the experimental bounds from the LHC, in the mass region that we consider. The lepton and fat jet in the signal and background have different features in their kinematic distributions, which we utilize for background reduction. The distribution of various kinematic variables has been 
shown in Figs. 3-6, both for the signal (for a sample mass point $M_{N}=900 \mathrm{GeV}$ ) and SM background. As can be seen from Fig. 3, the resulting lepton and the fat jet that originate from the decay of a heavy neutrino have a fairly large transverse momentum, with the peak occurring around $p_{T} \sim 400 \mathrm{GeV}$. On the other hand, the lepton and fat jet from background have a relatively lower $p_{T}$, as it is not originating from a very heavy state as signal. Therefore, the choice of high $p_{T}$ for the lepton and also for the fat jet removes a large fraction of the backgrounds. We divide our analysis into two separate segments: one for $M_{N}=600-900 \mathrm{GeV}$ and another for $M_{N}=1000-1200 \mathrm{GeV}$. The produced lepton and fat jet, therefore, have a relatively larger $p_{T}$. This motivates us to use a relatively strong cut on the charged lepton $p_{T}$ for $M_{N}=1000-1200 \mathrm{GeV}$, as compared to $M_{N}=600-900 \mathrm{GeV}$, and achieve better signal sensitivity.

In addition to the $p_{T}$ of the lepton and jet, we also use a strong cut on the pseudorapidity $\eta^{l}$ of the lepton. The

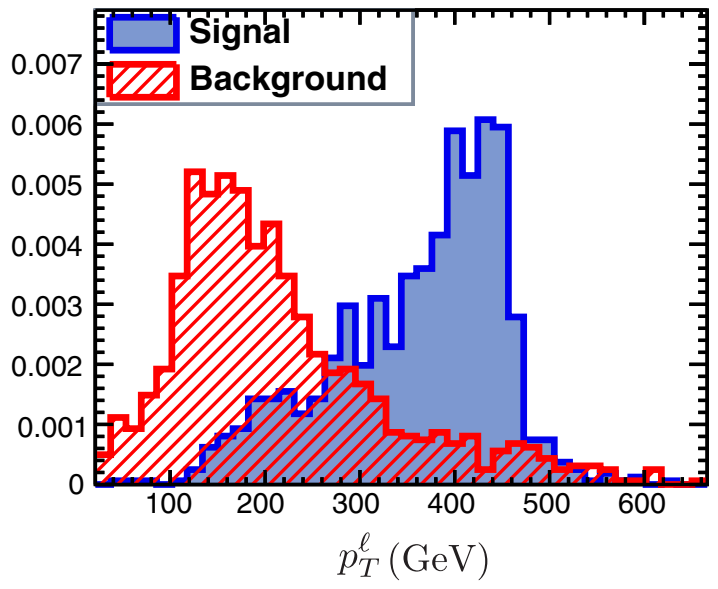

distribution of $\eta^{\ell}$ for signal and background, as can be seen from the left panel of Fig. 4, shows a sharp contrast. For the signal, the lepton is produced in the central region, while for the background, the peak occurs at $\eta^{\ell}$ far from zero. In the $e^{ \pm} \nu_{e} / \bar{\nu}_{e} j j$ background, the $W^{+} W^{-}$pair production contribution is large $(\sigma \sim 73 \mathrm{fb})$ as compared to the other contributions. For higher c.m. energy, $W^{+} W^{-}$pair produce more frequently along the beam line. This results in the noncentral feature of the lepton from the background.

In Fig. 4 (right panel), we show the $\Delta R$ separation between the charged lepton and the fat jet. For $\sqrt{s}=$ $1.4 \mathrm{TeV}$, the heavy neutrino of mass $M_{N}=900 \mathrm{GeV}$ does not have a very large momentum as compared to the case when $M_{N}$ has a smaller value. Therefore, the decay products of $N$ will have a large separation and the peak of $\Delta R$ occurs around $\Delta R(j, \ell) \sim 3.0$. For a smaller value of $M_{N}$, a heavy neutrino associates with a larger momentum. Hence the separation would be smaller, and the peak of $\Delta R(j, \ell)$ will

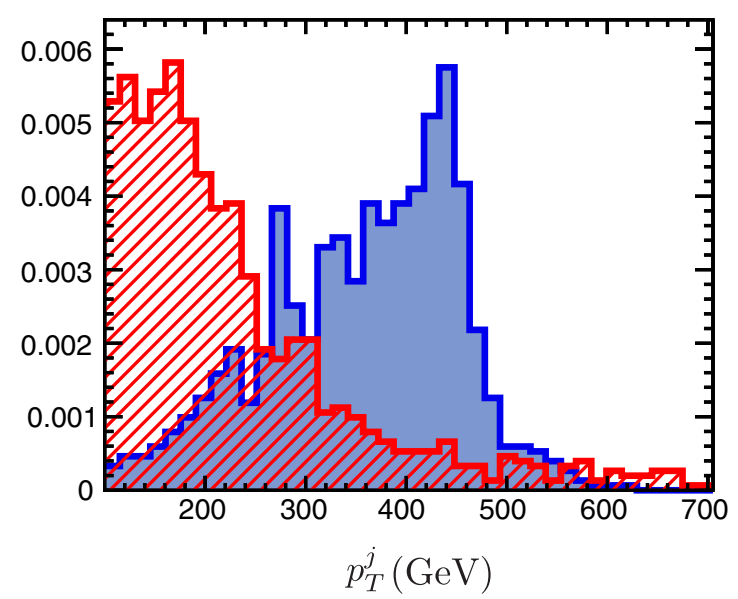

FIG. 3. The $p_{T}$ distribution of a lepton and fat jet for the heavy neutrino mass $M_{N}=900 \mathrm{GeV}$.
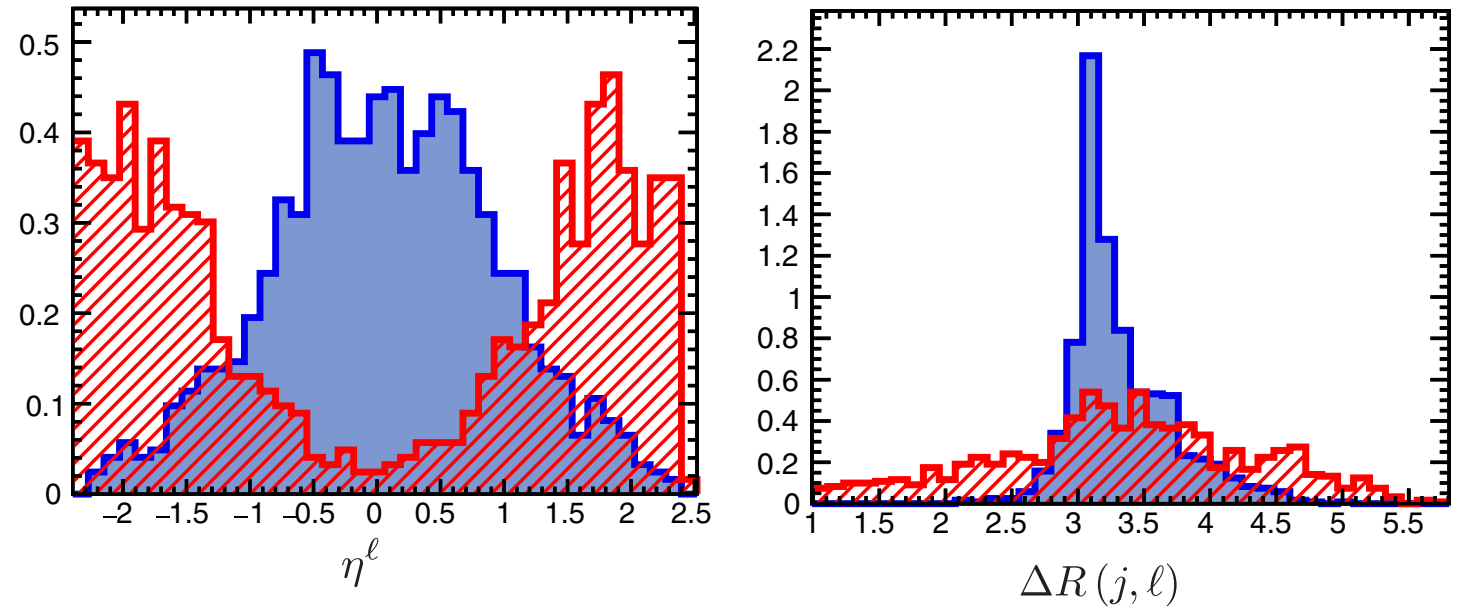

FIG. 4. The pseudorapidity $\eta^{\ell}$ distribution of the charge lepton (left panel) and $\Delta R$ separation between the jet and lepton (right panel) for the heavy neutrino mass $M_{N}=900 \mathrm{GeV}$. The peak in $\Delta R(j, \ell)$ for the background sample arises primarily due to the $W^{ \pm} W^{\mp}$ contribution. 

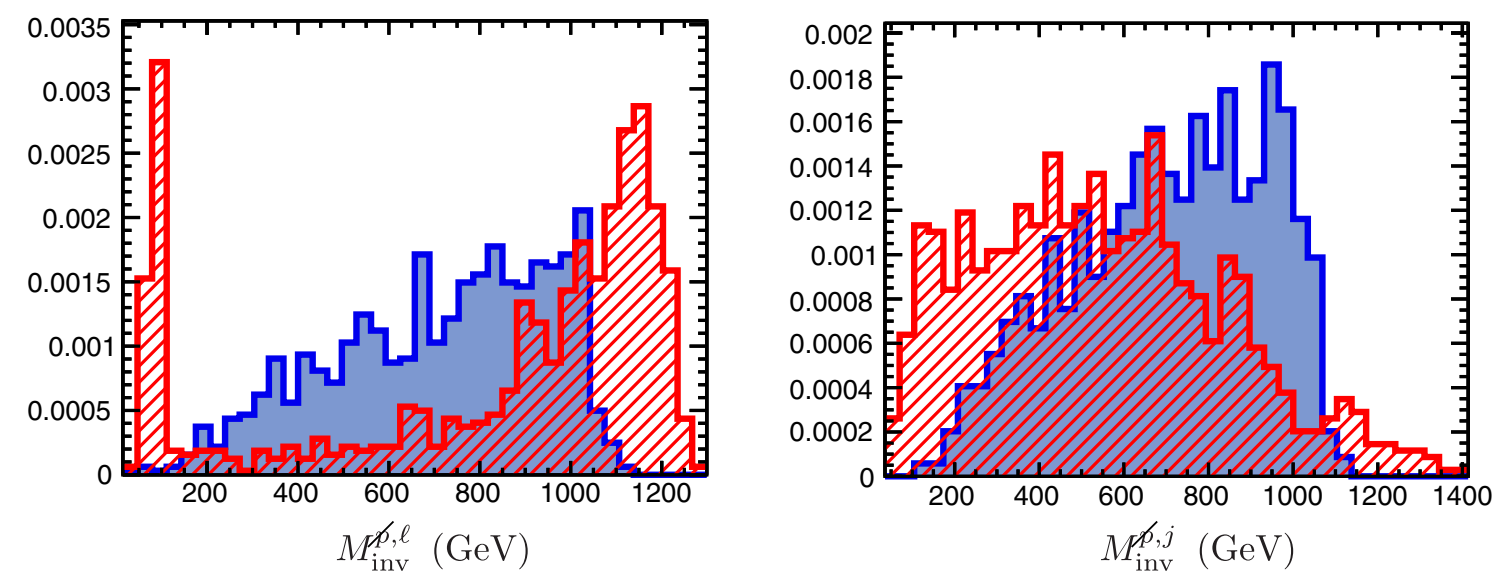

FIG. 5. The invariant mass distribution of the lepton and missing momenta $\not \not$ (left panel) and for the jet and missing momenta $\not \supset$ (right panel) for the heavy neutrino with mass $M_{N}=900 \mathrm{GeV}$.

shift towards smaller values. For the background, the separation between the lepton and fat jet arising from the $W^{+} W^{-}$sample is large. However, for other background contributions, this feature does not hold. Therefore, for the background, the peak of the $\Delta R$ distribution around $\Delta R(j, \ell)=3.0$ is smaller and primarily arises due to $W^{+} W^{-}$pair production. We implement a large separation cut between the jet and charge lepton to remove the background. For our mass choice, the lepton and fat jet are well separated, having a large $\Delta R(j, \ell)$.

We also use the information from the missing fourmomenta, $\not p$. Contrary to the hadron collider, the initial state four-momentum is completely known in an $e^{+} e^{-}$ machine. Balancing the final state visible particle's fourmomentum with the initial state four-momentum, one can construct the variable $\not p$. With this information, we construct a variable, namely, the invariant mass between $\not p$ and the lepton/jet. The invariant mass turns out to be large between the two four-momenta sets when their angular separation is large. Because of the large momentum in the relatively lighter $M_{N}$, the produced $W$ 's will be aligned along the direction of $N$. Therefore, for lower $M_{N}$, the angular separation between the $\not p$ and jet originating from $W$ decay is large, which results in a larger invariant mass $M_{\text {inv }}^{\not, j}$. As a result, we implement a higher cut on $M_{\mathrm{inv}}^{\not, j}$ for relatively lower $M_{N} \sim 600-900 \mathrm{GeV}$ as compared to the higher mass range 1000-1200 GeV. We note in passing that $M_{\text {inv }}^{p, \ell}$ also possesses a similar feature. However, we implement the same cut for the entire mass range. For the background distribution, the invariant mass $M_{\mathrm{inv}}^{p, \ell}$ has another peak near $80 \mathrm{GeV}$, which occurs primarily due to the $W^{+} W^{-}$contribution. For the signal, $E_{T}$ is large for a relatively lower $M_{N}$. We show the distribution in Fig. 6 . We demand $E_{T}<150.0$ throughout our analysis. Below we list different cuts that we implement. We have mildly optimized our cuts for the two different mass regions $M_{N}=$ 600-900 GeV (referred to as CBA-I) and 1000-1200 GeV (referred to as CBA-II) for the cut-based analysis. The cuts are constructed in such a way that we achieve the best signal significance.

CBA-I for $M_{N}=600-900 \mathrm{GeV}$

(i) $C 1$ : Transverse momentum for $e^{ \pm}: p_{T}>200 \mathrm{GeV}$.

(ii) $C 2$ : Transverse momentum of the fat jet: $p_{T}>$ $200 \mathrm{GeV}$.

(iii) C3: Transverse missing energy: $E_{T}<150.0 \mathrm{GeV}$.

(iv) C4: Pseudorapidity of $e^{ \pm}:-1.0<\eta^{\ell}<1.0$.

(v) C5: Jet-lepton separation: $2.8<\Delta R(j, \ell)<3.8$.

(vi) C6: Invariant mass of $\not \supset$ and lepton: $150<M_{\mathrm{inv}}^{p, \ell}<$ $950 \mathrm{GeV}$.

(vii) $C 7$ : Invariant mass of $\not \not$ and jet: $M_{\text {inv }}^{p, j}>600.0 \mathrm{GeV}$. We again optimize the cuts in a different mass window as

CBA-II for $M_{N}=1000-1200 \mathrm{GeV}$

(i) Cl: Transverse momentum for $e^{ \pm}: p_{T}>350 \mathrm{GeV}$.

(ii) $C 2$ : Transverse momentum of the fat jet: $p_{T}>$ $350 \mathrm{GeV}$.

(iii) C3: Transverse missing energy: $E_{T}<150.0$.

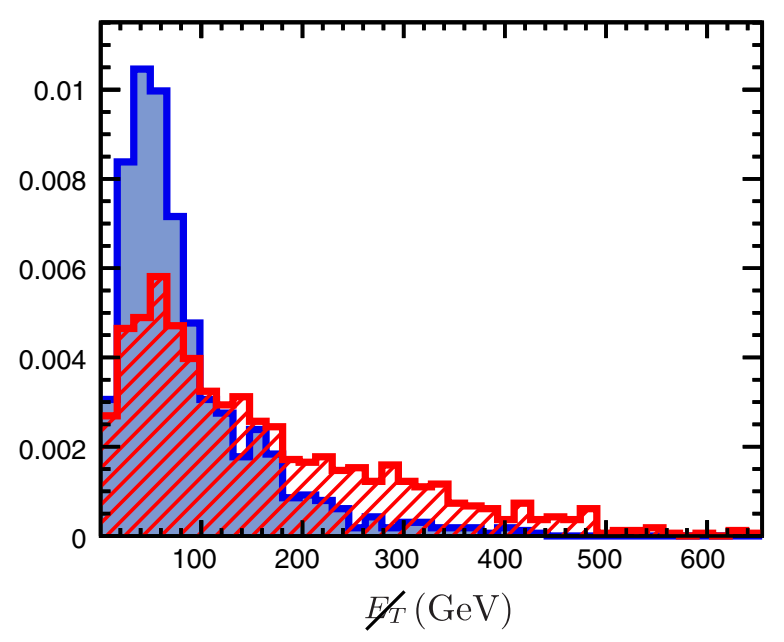

FIG. 6. The missing transverse energy $\mathscr{E}_{T}$ distribution for heavy neutrino mass $M_{N}=900 \mathrm{GeV}$. 
(iv) $C 4$ Pseudorapidity of $e^{ \pm}:-1.0<\eta^{\ell}<1.0$.

(v) C5: Jet-lepton separation: $2.8<\Delta R(j, \ell)<3.8$.

(vi) $C 6$ : Invariant mass of $\not p$ and lepton: $150<M_{\text {inv }}^{\not p, \ell}<$ $950 \mathrm{GeV}$.

(vii) $C 7$ : Invariant mass of $\not p$ and jet: $M_{\text {inv }}^{p, j}>400.0 \mathrm{GeV}$.

Below, we discuss in detail heavy neutrino searches for $\sqrt{s}=3 \mathrm{TeV}$.

\section{2. $M_{N}=1300-2700 \mathrm{GeV}$ with $\sqrt{s}=3 \mathrm{TeV}$}

Heavy neutrinos in the multi-TeV mass range can be probed with a higher c.m. energy. As an example, we consider $\sqrt{s}=3 \mathrm{TeV}$, relevant for CLIC, and present our analysis for the mass range $M_{N}=1300-2700 \mathrm{GeV}$. Similar to the previous analysis, here we use slightly different cuts for $M_{N}=1300-1900$ and $2100-2700 \mathrm{GeV}$. The same set of cuts cannot be used for the entire mass range, as the kinematics of the final states for $2700 \mathrm{GeV}$ are widely different from $1300 \mathrm{GeV}$. There are a few variables that we have taken as common though for both of the regions. These are the electron $p_{T}$; the difference of the pseudorapidity between the jet and $\not p, \Delta \eta(j, p)$; the invariant mass of the lepton and $\not p, M_{\text {inv }}^{\not p, \ell}$; and the invariant mass of the jet and $\not p, M_{\mathrm{inv}}^{\not, j}$. We show the distributions of various kinematic variables in Figs. 7 and 8.

For the mass range $2100-2700 \mathrm{GeV}$, the electron $e^{ \pm}$ from $N$ decay will have a very high momentum. Therefore, with stringent cuts on the lepton momentum, the background becomes negligible. We show the distribution for the $p_{T}$ of lepton in Fig. 7 for the heavy neutrino mass $M_{N}=2.1 \mathrm{TeV}$. We choose a lower $p_{T}$ cut on electron $p_{T}$ for $M_{N}=1300-1900 \mathrm{GeV}$ and larger for the higher mass case. The reason is similar to that mentioned for the $1.4 \mathrm{TeV}$ analysis in Sec. III A 1.

In the right panel of Fig. 7, we show the distribution of pseudorapidity separation between the fat jet and $\not p$. The separation is large for large angular separation. For a
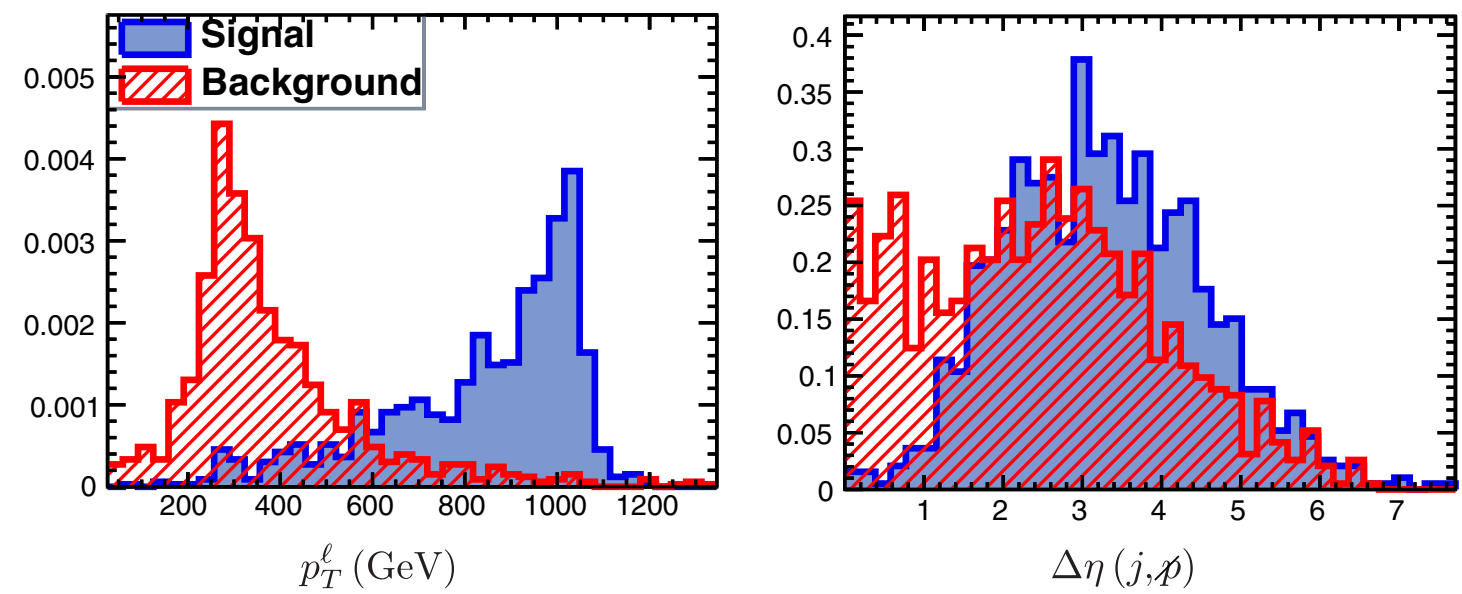

FIG. 7. The $p_{T}$ distribution of the charged lepton (left panel) and pseudorapidity separation between the jet and $\not p$ (right panel) for the heavy neutrino mass $M_{N}=2100 \mathrm{GeV}$.
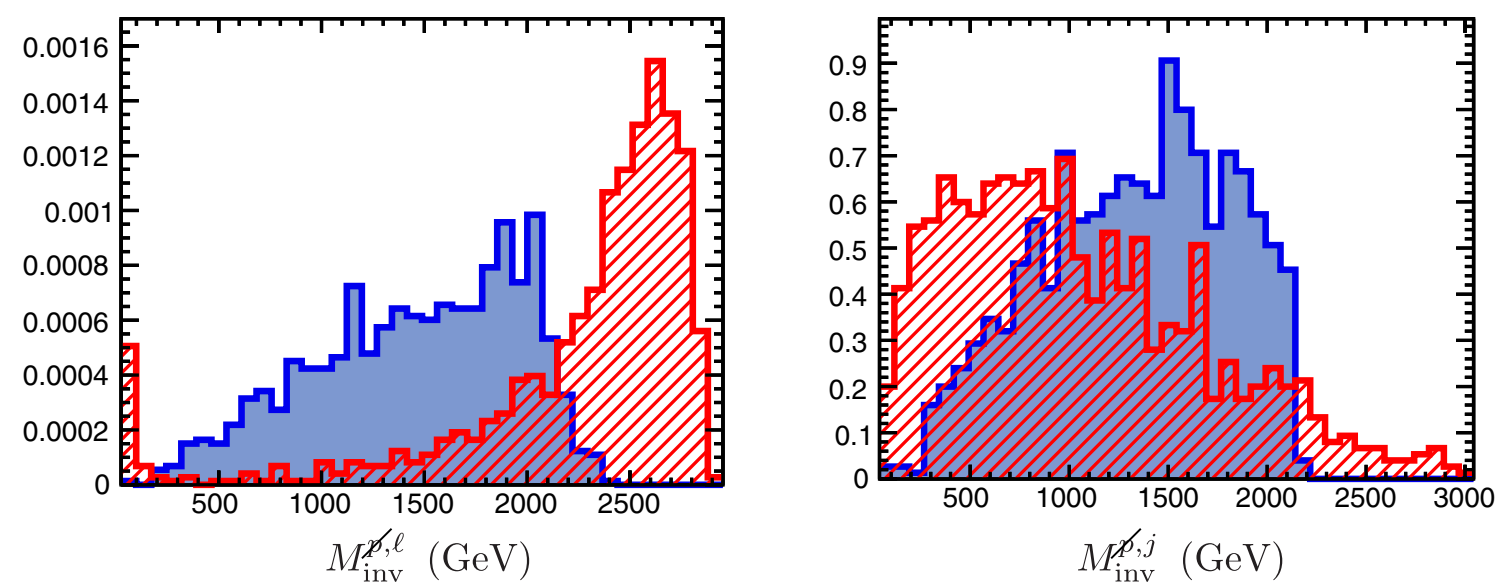

FIG. 8. The invariant mass distribution of the charged lepton and $\not \supset$ (left panel) and invariant mass distribution between the jet and $\not \supset$ (right panel) for the heavy neutrino mass $M_{N}=2100 \mathrm{GeV}$. 
relatively lighter $N$, it is more likely that the produced fat jet and $\not p$ have a significant angular separation between them. Therefore, we implement a large cut on $\Delta \eta(j, \not \supset)$ for the 1300-1900 GeV mass range compared to the 2100$2700 \mathrm{GeV}$ range. For a $2100 \mathrm{GeV}$ mass the peak occurs around $\Delta \eta(j, \not \supset)=3.0$. In the background, the $W^{+} W^{-}$ sample results in a peak around $\Delta \eta(j, \not \supset)=3.0$. However, the background also has other contributions that result in smaller separation $\Delta \eta(j, \not \supset)$. Overall the background is more likely to have less angular separation as compared to the signal. The invariant mass distributions for $3 \mathrm{TeV}$, such as $M_{\text {inv }}^{\not, \ell}$ and $M_{\text {inv }}^{\not, j}$, have similar features as for $1.4 \mathrm{TeV}$. Therefore, we implement a strong cut on these variables for a relatively lighter $N$ mass. Also, $\Delta \phi(j, \not p)$ is almost uniformly distributed for the background, whereas the signal has a larger cross section in the small $\Delta \phi(j, \not \supset)$ region. Therefore, to enhance the signal sensitivity, we reject events with $\Delta \phi(j, \not \supset)>2.0$ as shown in the left panel of Fig. 9. An additional variable that we particularly use for the 2100 $2700 \mathrm{GeV}$ mass range is the jet mass. For the signal, the jet mass $\left(\mathrm{M}_{\mathrm{J}}\right)$ has a peak near the $W$ boson mass (see right panel of Fig. 9) as the signal jets are coming from the boosted $W$ boson. The background also has a similar peak around the $W$ boson mass, since $W^{+} W^{-}$pair production contributes significantly in background. However, the $W$ boson in the background is relatively less boosted as compared to the signal, as this is not generated from the decay of a heavy resonance. This results in a broad peak for the background compared to the signal. We choose a window on the jet mass $\left(\mathrm{M}_{\mathrm{J}}\right)$ variable as 70-90 GeV. Below, we list all the cuts that we implement. Similar to the previous case, the final state contains one isolated lepton $e^{ \pm}$, one fat-jet $j_{\text {fat }}$ with radius $R=1.0$, and missing four-momentum $\not p$.

CBA-III for $M_{N}=1300-1900 \mathrm{GeV}$

(i) D1: $p_{T}$ for electron $p_{T}>450 \mathrm{GeV}$.

(ii) D2: Pseudorapidity of $e^{ \pm}:-1.0<\eta^{\ell}<1.0$.

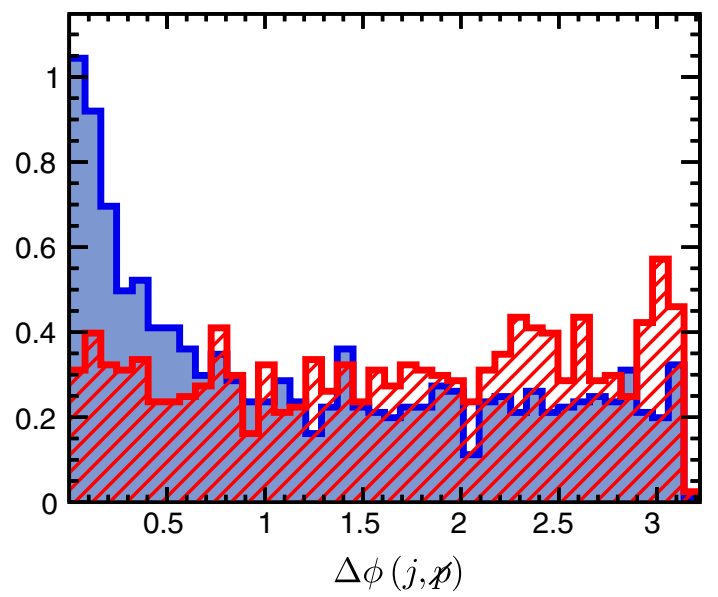

(iii) $D 3$ : Jet- $\not p$ rapidity separation $\Delta \eta(j, \not \supset): \Delta \eta(j, \not \supset)>3.0$.

(iv) $D 4$ : Jet-lepton rapidity separation $\Delta \eta(j, \ell)$ : $\Delta \eta(j, \ell)<2.0$.

(v) D5: Invariant mass of $\not \not$ and lepton: $200<M_{\mathrm{inv}}^{p, \ell}<$ $2500 \mathrm{GeV}$.

(vi) D6: Invariant mass of $\not p$ and jet: $M_{\mathrm{inv}}^{\not p, j}>1300.0 \mathrm{GeV}$.

CBA-IV for $M_{N}=2100-2700 \mathrm{GeV}$

(i) DI: $p_{T}$ for electron: $p_{T}>600 \mathrm{GeV}$.

(ii) D2: Missing transverse energy: $E_{T}<200.0 \mathrm{GeV}$.

(iii) D3: Jet- $\not p$ rapidity separation $\Delta \eta(j, \not p): \Delta \eta(j, p)>0.5$.

(iv) $D 4$ : Jet- $\not \supset$ azimuthal angle separation $\Delta \phi(j, \not \supset)$ : $\Delta \phi(j, \not \supset)<2.0$.

(v) D5: Invariant mass of $\not p$ and lepton: $200<M_{\mathrm{inv}}^{\not, \ell}<$ $2000 \mathrm{GeV}$.

(vi) D6: Invariant mass of $\not p$ and jet: $200.0<M_{\mathrm{inv}}^{p, j}<$ 2000.0 GeV.

(vii) D7: Jet mass $M_{J}: 80.0<M_{J}<90.0 \mathrm{GeV}$.

Before going into the details of signal and background efficiencies with the full cut-based analysis, we discuss the important issues pertaining to MVA and also present a comparative study between the two methods. After a detailed discussion about the multivariate analysis, we will discuss the results. We also project out the required luminosity to obtain a discovery significance.

\section{B. Multivariate analysis}

We optimize our search strategy and show the importance of our chosen variables by performing a multivariate analysis using the boosted decision tree (BDT) algorithm. This is implemented within the ROOT framework for the Toolkit for Multivariate Analysis (TMVA). In order to classify a set of data, a binary structured decision tree takes a yes/no decision on one single variable at a time until some stop criterion is satisfied. Obviously, the classification is whether the data are signal-like or backgroundlike. For example, in our case, the tree starts with a root node and uses variables such as $p_{T}^{\ell}, p_{T}^{j}, M_{\mathrm{int}}^{p, j}, M_{\mathrm{int}}^{p, \ell}, E_{T}^{\epsilon}, \eta^{\ell}$, and so on

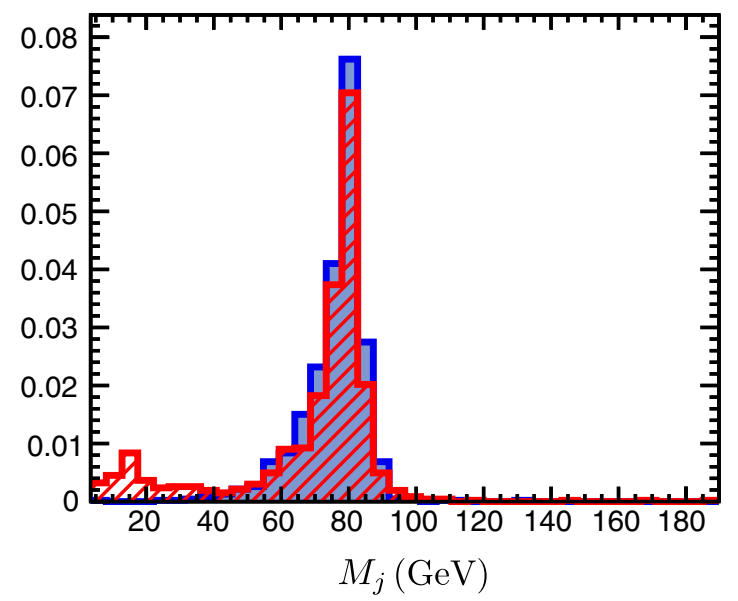

FIG. 9. The azimuthal separation between the jet and $\not \supset$ (left panel) and distribution of the jet mass (right panel) for the heavy neutrino mass $M_{N}=2100 \mathrm{GeV}$. 
to segregate the data into signal-like or backgroundlike. A variety of separation criteria can be used to discriminate between the signal and background events. Perhaps the most common is the Gini index defined by $p(1-p)$, where $p$ is the purity of the sample. This iteration stops when the maximum separation between the signal and background samples is achieved. Extending this concept from one tree to several trees, which eventually forms a forest (random forest), is called boosting. This is extremely important as the outcome of a single decision tree is susceptible to statistical fluctuations. Boosting helps to reduce such errors by giving a larger weight to the misclassified events for the next iteration. Ultimately, the majority vote among the trees in the random forest is taken to classify the events.

For our work, we choose the BDT parameters as NTrees or the number of trees in a forest to be 400 .

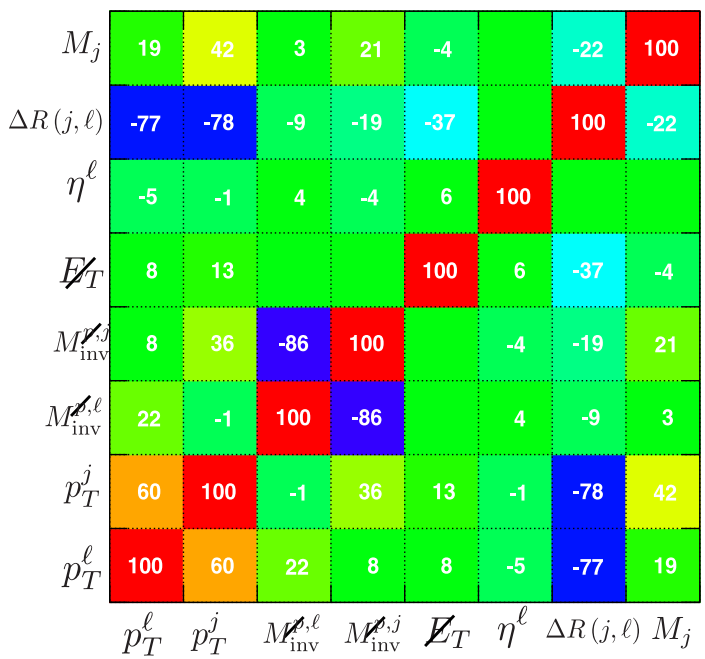

The maximum depth of the decision tree is considered to be MaxDepth=5 and the minimum percentage of training events required in a leaf node is taken as MinNodeSize $=2.5 \%$. For boosting the decision tree, we consider the AdaBoost method and the corresponding learning rate for the AdaBoost algorithm is taken to be AdaBoostBeta=0.5. We also present correlation plots as well as BDT responses using TMVA in Figs. 10 and 11 , respectively. The correlation between any two random variables used in our analysis (say, $X$ and $Y$ ) is defined as

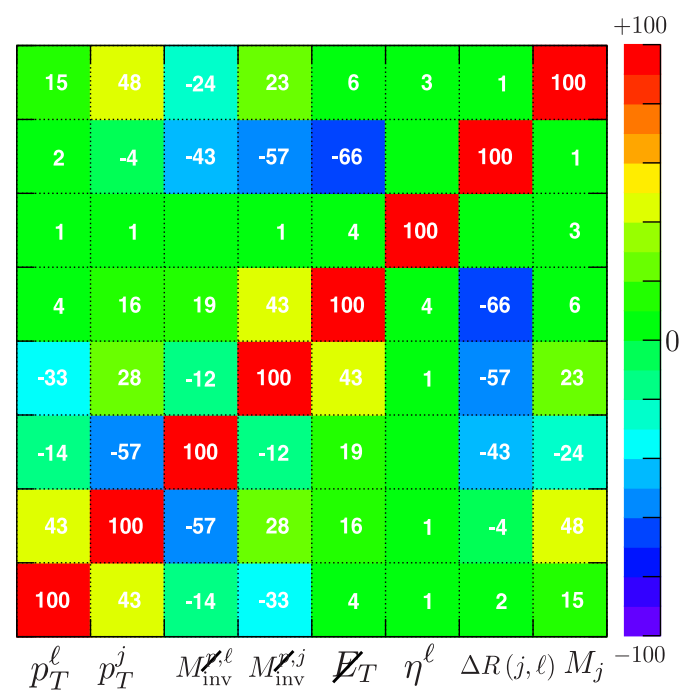

FIG. 10. The plot in the left panel depicts correlation for the used variables for signal events while the plot in the right panel is for background events. We consider $\sqrt{s}=1.4 \mathrm{TeV}$.

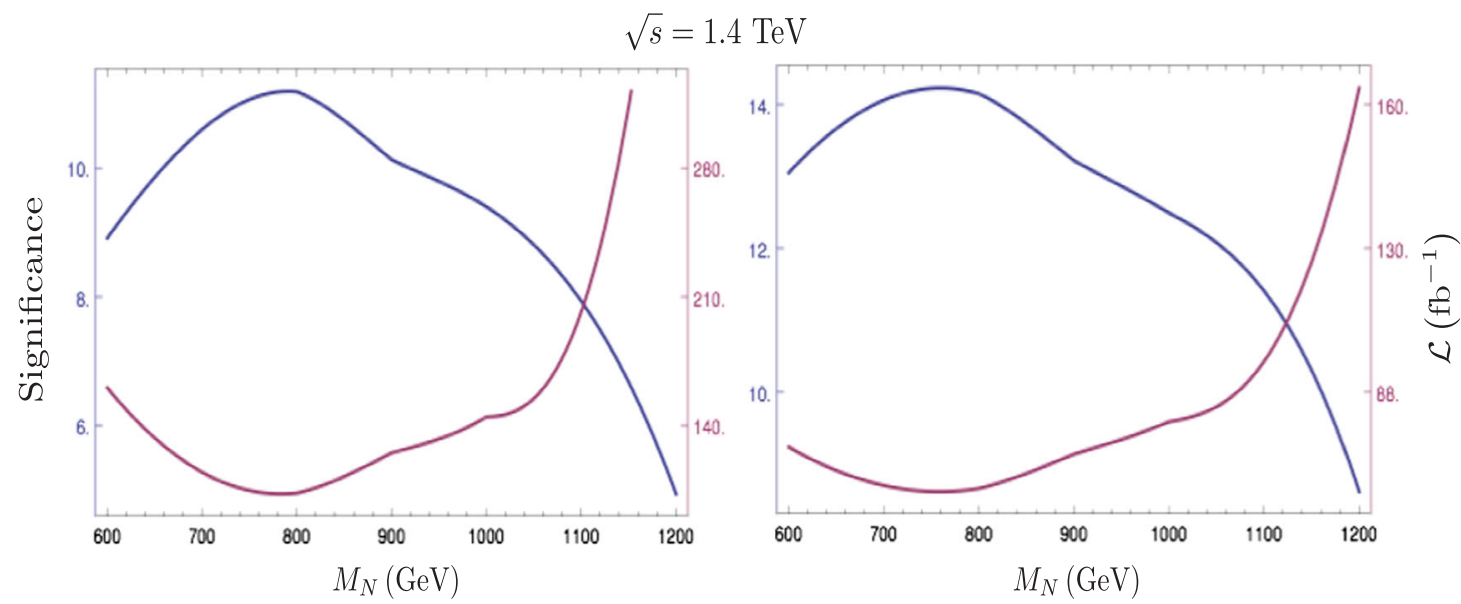

FIG. 11. The blue and purple lines correspond to the signal significance and required luminosity for $5 \sigma$ significance, respectively. Left: Variation of signal significance vs mass of the heavy neutrino using the cut-based analysis at $\sqrt{s}=1.4 \mathrm{TeV}$. Right: Same plot as left using BDT. The active-sterile mixing has been considered $\left|V_{e N}\right|=0.01$. 
where $\sigma$ is the usual standard deviation of the input variables and $\operatorname{cov}(X, Y) \equiv E(X Y)-E(X) E(Y)$. It is rather conspicuous that $\rho=0$ would imply independent variables. Usually, the more independent the variables are, the more information they carry and therefore the more they help to distinguish between signal and background events. To quantify the performance of each variable, the relative ranking among the variables is given as (i) $M_{\mathrm{inv}}^{p, \ell}$, (ii) $M_{\mathrm{inv}}^{p, j}$, (iii) $p_{T}^{\ell}$, (iv) $p_{T}^{j}$, (v) $E_{T}$, (vi) $\eta^{\ell}$, (vii) $\eta^{j}$, and finally (viii) $\Delta R(j, \ell)$. These rankings of performance of the chosen variables may not always be obvious from the distribution plots shown in Figs. 3-6. Hence, ranking of the input variables is obtained based on how often these variables are used to split the decision trees. The BDT output describes a mapping between the $n$-dimensional phase space of our chosen variables to one-dimensional variables. In general, any specific value of the BDT variable can be chosen as a cut; however, a particular cut value in the BDT output corresponds to maximum signal purity and consequently, maximum signal significance. We have also compared our results with the commonly used cutbased analysis with the state-of-the-art multivariate analysis. Obviously, significant enhancement in both signal purity and signal significance can be achieved by using MVA.

\section{Signal and background efficiency}

We divide the discussion of this section into two categories. Firstly, the signal and background significance for $\sqrt{s}=1.4 \mathrm{TeV}$ is discussed, followed by the discussion for $3 \mathrm{TeV}$ c.m. energy. We also compare our results from both the cut-based and multivariate analysis.

\section{Signal and background efficiency for $\sqrt{s}=1.4 \mathrm{TeV}$}

As a benchmark, we show the gradual change in the cross section in Table I after implementing the cuts as discussed earlier.

In Tables III and IV, the second column corresponds to the partonic cross section $\left(\sigma_{\text {partonic }}\right)$ for $e^{ \pm}+j j+\not p$. The third column represents the cross section after all the cuts, where we also include the detector effect. For the mass range $600-1200 \mathrm{GeV}$, the partonic cross section varies from $\sigma_{\text {partonic }} \sim 2.39-0.8 \mathrm{fb}$. After taking into account all the above-mentioned cuts and detector effects, the cross section drops nominally by a factor of $\sigma_{D} / \sigma_{\text {partonic }} \sim 2-3$.
For comparatively lower masses, such as $600 \mathrm{GeV}$, the drop is relatively larger. This happens because for relatively lower $M_{N}$, the decay products $W^{ \pm}$and charge lepton $l^{\mp}$ have smaller $p_{T}$ as compared to the higher $M_{N}$ scenario. A high $p_{T} W^{ \pm}$-boson has a larger probability to make a fat jet compared to the low $p_{T} W^{ \pm}$-boson. Therefore, for higher $M_{N}$, the cuts reduce the signal cross section nominally. On the other hand, the background cross section $\sigma_{\mathrm{BKG}} \sim 750 \mathrm{fb}$ at the partonic level falls drastically, $\sigma_{\mathrm{BKG}} \sim 1.86 \mathrm{fb}$, after all the cuts. In particular, we stress that the choice of a high $p_{T}$ for the lepton and jet kills almost all of the backgrounds. For a $t \bar{t}$ background with 1.4 and $3 \mathrm{TeV}$ c.m. energy, the partonic cross section is 23.34 and $5.18 \mathrm{fb}$, respectively (considering the semileptonic decay of $t \bar{t}$ ). Applying a b-jet veto [104] and other selection cut criteria, this background has a minuscule contribution to our final results. Although most of the resonant and nonresonant backgrounds are included in our estimation, in Table II we list few of the resonant backgrounds. For example, the $t \bar{t}$, $W^{+} W^{-}\left(W^{+} \rightarrow \tau^{+} \nu_{\tau}, W^{-} \rightarrow \ell^{-} \nu_{\ell}\right), \quad Z Z\left(Z \rightarrow e^{+} e^{-}, Z \rightarrow j j\right)$ backgrounds are not significant after applying the selection cuts. The effect of the beam induced photon to our jet counting is negligible because the beam induced background $\gamma \gamma \rightarrow$ hadrons has a lower production rate (10\%) [105-107] in the central region (barrel) of the detector. The hadrons coming from the beam induced photon are mostly in the forward direction of the detector (90\%) [105-107]. In our case the jet is produced in the central region of the detector $\left(\left|j_{\text {eta }}\right|<2.0\right)$. Also the effect of the $\gamma \gamma \rightarrow$ hadrons background on the missing energy measurement is very negligible for CLIC energy [107].

The signal sensitivity can be computed using the following expression:

$$
n_{s}=\frac{S_{d}}{\sqrt{S_{d}+B_{d}}}
$$

where $S_{d}$ and $B_{d}$ represent the signal and background event numbers after all the cuts and detector effect. We show the signal sensitivity in the fourth and fifth column of Tables III, and IV. For both the lower and higher masses, the significance is lower and peaks in the middle region. For a lower mass of $N$, the cross section is larger and for a higher mass the cross section is smaller. However, the cut

TABLE I. Partonic cross section and the cross section after each of the cuts for illustrative signal mass point $M_{N}=900 \mathrm{GeV}$. We also show the background cross section.

\begin{tabular}{lccccccc}
\hline \hline Mass $(\mathrm{GeV})$ & \multicolumn{7}{c}{ Cross sections at the partonic level and after cuts } \\
\hline \multirow{2}{900}{} & $\sigma_{\text {partonic }}(\mathrm{fb})$ & $\mathrm{C} 1+\mathrm{C} 2$ & $\mathrm{C} 3$ & $\mathrm{C} 4$ & $\mathrm{C} 5$ & $\mathrm{C} 6$ & $\mathrm{C} 7$ \\
Background & 1.78 & 1.24 & 1.01 & 0.88 & 0.85 & 0.83 & 0.73 \\
\hline \hline
\end{tabular}


TABLE II. Cross-section list of the resonant backgrounds.

\begin{tabular}{lrrrcc}
\hline \hline & $\sigma$ at $1.4 \mathrm{TeV}(\mathrm{fb})$ & & $\sigma$ at $3 \mathrm{TeV}(\mathrm{fb})$ \\
\cline { 2 - 3 } Backgrounds & Partonc & $\begin{array}{c}\text { After } \\
\text { cuts }\end{array}$ & & Partonic & $\begin{array}{c}\text { After } \\
\text { cuts }\end{array}$ \\
\hline$e^{+} e^{-} \rightarrow e^{+} \nu_{e} j j$ & 751.45 & 1.86 & 472.5 & 0.91 \\
$e^{+} e^{-} \rightarrow W^{+} W^{-} \rightarrow e^{+} \nu_{e} \tau^{-} \nu_{\tau}$ & 7.01 & $\sim 0.0$ & 1.5 & $\sim 0.0$ \\
$e^{+} e^{-} \rightarrow Z Z \rightarrow e^{+} e^{-} j j$ & 0.77 & $\sim 0.0$ & $7.5 \times 10^{-5}$ & $\sim 0.0$ \\
$e^{+} e^{-} \rightarrow t \bar{t} \rightarrow$ semi-leptonic & 23.34 & $\sim 0.0$ & 5.18 & $\sim 0.0$ \\
\hline \hline
\end{tabular}

TABLE III. Cross section for signal and background in $\mathrm{fb}$. We also show the significance for luminosity $500 \mathrm{fb}^{-1}$.

\begin{tabular}{lcccccc}
\hline \hline \multicolumn{3}{c}{ Mass and cross section } & & \multicolumn{2}{c}{ Significance } \\
\cline { 1 - 3 } Mass $(\mathrm{GeV})$ & $\sigma_{\text {partonic }}(\mathrm{fb})$ & $\sigma_{D}(\mathrm{fb})$ & & CBA-I & BDT \\
\hline 600 & 2.39 & 0.63 & & 8.92 & 13.05 \\
700 & 2.24 & 0.77 & & 10.61 & 14.06 \\
800 & 2.03 & 0.82 & & 11.20 & 14.15 \\
900 & 1.78 & 0.73 & & 10.14 & 13.22 \\
Background & 751.42 & 1.86 & & $\ldots$ & $\cdots$ \\
\hline \hline
\end{tabular}

TABLE IV. Cross section for signal and background in $\mathrm{fb}$. We also show the significance for luminosity $500 \mathrm{fb}^{-1}$.

\begin{tabular}{lccccc}
\hline \hline \multicolumn{3}{c}{ Mass and cross section } & & \multicolumn{2}{c}{ Significance } \\
\cline { 1 - 2 } \cline { 6 - 7 } Mass $(\mathrm{GeV})$ & $\sigma_{\text {partonic }}(\mathrm{fb})$ & $\sigma_{D}(\mathrm{fb})$ & & CBA-II & BDT \\
\hline 1000 & 1.49 & 0.62 & & 9.41 & 12.49 \\
1100 & 1.16 & 0.51 & & 7.94 & 11.41 \\
1200 & 0.80 & 0.30 & & 4.93 & 8.61 \\
Background & 751.42 & 1.55 & & $\cdots$ & $\cdots$ \\
\hline \hline
\end{tabular}

efficiency is low for small masses, which results in the drop of the signal cross section. The fall of the cross section and sensitivity in the higher mass regime occurs due to the smaller partonic cross section. The significance curve for BDT and the cut-based analysis have similar features.

\section{Signal and background efficiency for $\sqrt{s}=3 \mathrm{TeV}$}

We discuss the results obtained using both the cut-based analysis and MVA for $\sqrt{s}=3 \mathrm{TeV}$. The cross section for the signal and the background is given in Tables VI and VII, for the mass ranges $1300-1900$ and $2100-2700 \mathrm{GeV}$, respectively. Similar to the previous analysis, the second and third columns represent the partonic cross section and the cross section after all the cuts. For the above-mentioned mass range, the partonic cross section varies from $\sigma_{\text {partonic }} \sim 2.48-0.60 \mathrm{fb}$. The background cross section for $\sqrt{s}=3 \mathrm{TeV}$ c.m. energy is $\sigma_{\mathrm{BKG}}=472.36 \mathrm{fb}$ at the partonic level and drops down to the sub-fb level after all the cuts. For the signal, the effect of the cuts is nominal,
TABLE V. Partonic cross section and the cross section after each of the cuts for the illustrative signal mass point $M_{N}=2100 \mathrm{GeV}$. The cross section for the background is also shown.

\begin{tabular}{lccccccccc}
\hline \hline Mass $(\mathrm{GeV})$ & \multicolumn{6}{c}{ Cross sections at the partonic level and after cuts } \\
\hline \multirow{2}{*}{2100} & $\sigma_{\text {partonic }}(\mathrm{fb})$ & $\mathrm{D} 1$ & $\mathrm{D} 2$ & $\mathrm{D} 3$ & D4 & D5 & D6 & D7 \\
& 1.61 & 0.91 & 0.76 & 0.76 & 0.54 & 0.49 & 0.47 & 0.38 \\
Background & 472.5 & 27.29 & 7.02 & 5.95 & 3.57 & 1.89 & 1.70 & 1.38 \\
\hline \hline
\end{tabular}

TABLE VI. Cross section for signal and background in $\mathrm{fb}$. We show the significance for luminosity $500 \mathrm{fb}^{-1}$.

\begin{tabular}{lccccc}
\hline \hline \multicolumn{3}{c}{ Mass and cross section } & & \multicolumn{2}{c}{ Significance } \\
\cline { 1 - 2 } Mass $(\mathrm{GeV})$ & $\sigma_{\text {partonic }}(\mathrm{fb})$ & $\sigma_{D}(\mathrm{fb})$ & & CBA-III & BDT \\
\hline 1300 & 2.48 & 0.78 & & 13.41 & 16.15 \\
1500 & 2.33 & 0.81 & & 13.81 & 18.61 \\
1700 & 2.12 & 0.71 & & 12.47 & 19.60 \\
1900 & 1.89 & 0.55 & & 10.17 & 17.89 \\
Background & 472.5 & 0.91 & & $\ldots$ & $\cdots$ \\
\hline \hline
\end{tabular}

TABLE VII. Cross section for signal and background in $\mathrm{fb}$. We show the significance for luminosity $500 \mathrm{fb}^{-1}$.

\begin{tabular}{lccccc}
\hline \hline \multicolumn{3}{c}{ Mass and cross section } & & \multicolumn{2}{c}{ Significance } \\
\cline { 1 - 3 } \cline { 6 - 7 } Mass $(\mathrm{GeV})$ & $\sigma_{\text {partonic }}(\mathrm{fb})$ & $\sigma_{D}(\mathrm{fb})$ & & CBA-IV & BDT \\
\hline 2100 & 1.61 & 0.38 & & 6.40 & 16.53 \\
2300 & 1.31 & 0.36 & & 6.10 & 16.46 \\
2500 & 0.97 & 0.27 & & 4.70 & 14.99 \\
2700 & 0.60 & 0.17 & & 3.05 & 10.86 \\
Background & 472.5 & 1.38 & & $\ldots$ & $\cdots$ \\
\hline \hline
\end{tabular}

reducing the cross section to $\sigma_{D}=0.78-0.17 \mathrm{fb}$. A detailed cut efficiency is presented in Table V, for the illustrative signal sample $M_{N}=2100 \mathrm{GeV}$ and also for the background.

In Figs. 11 and 12, we show the variation of signal sensitivity (blue line) and required luminosity (purple line) for $5 \sigma$ significance with mass $M_{N}$. Both the figures have similar features. For a lower value of $M_{N}$, the cut efficiency is low, which results in a smaller signal cross section and reduced sensitivity. For a higher mass, the reduction occurs due to the lower partonic cross section. The signal significance reaches its maximum in the mid region. We also show the required luminosity to achieve $5 \sigma$ significance in the same plot. We emphasize that a heavy neutrino in the mass range $M_{N}=600-1100 \mathrm{GeV}$ can be discovered with $\mathcal{L} \leq 100 \mathrm{fb}^{-1}$ of data in the $\sqrt{s}=1.4 \mathrm{TeV}$ run of CLIC. For the c.m. energy $3 \mathrm{TeV}$, the required luminosity to probe $M_{N}=1300-2300 \mathrm{GeV}$ is $\mathcal{L}=50 \mathrm{fb}^{-1}$. For the previous discussions, we considered a benchmark value for 

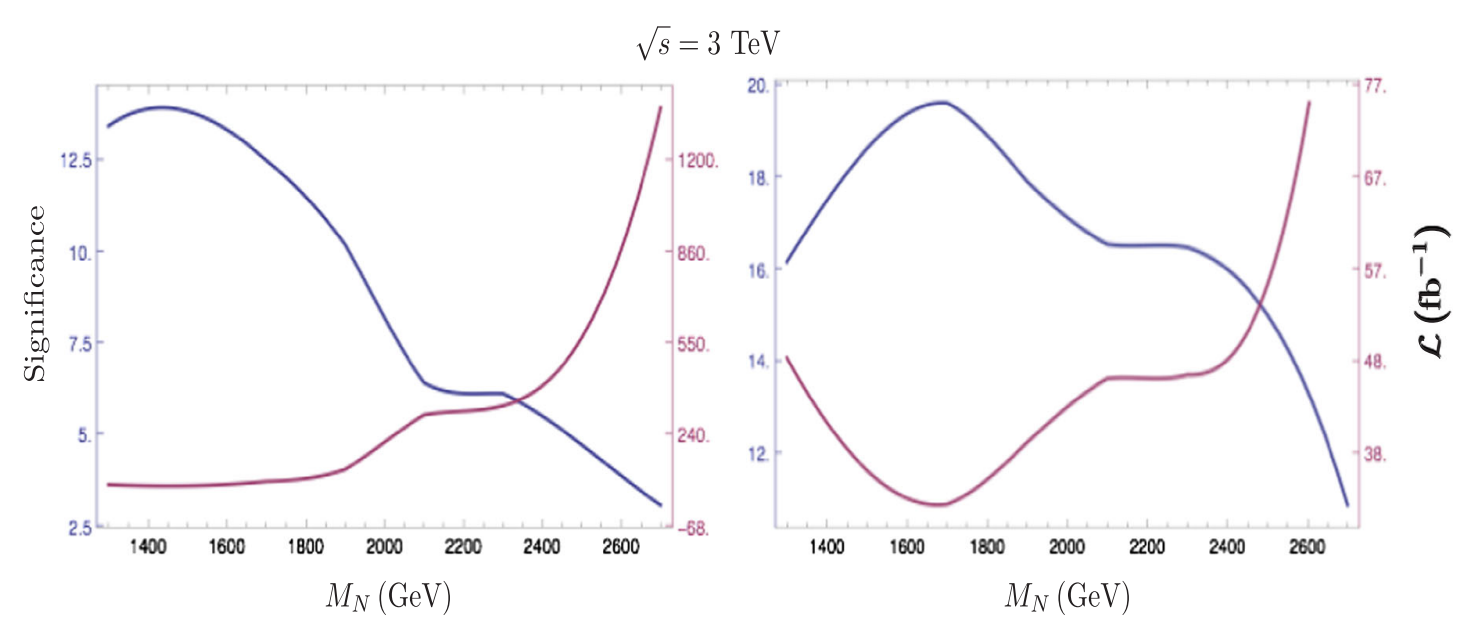

FIG. 12. The blue and purple lines correspond to the signal significance and required luminosity for $5 \sigma$ significance, respectively. Left: Signal significance and required luminosity vs mass of the heavy neutrino using the cut-based analysis at $E_{\mathrm{cm}}=3 \mathrm{TeV}$. Right: Same plot as left using BDT. The active-sterile mixing has been considered $\left|V_{e N}\right|=0.01$.

the active-sterile mixing $\left|V_{e N}\right|=0.01$. The cross section for the heavy neutrino production varies quadratically with the mixing. Hence, using Eq. (3.2), the bound on the activesterile mixing can be obtained as follows:

$$
n_{s}=\frac{\sigma_{s}^{0}\left|V_{e N}\right|^{2} \sqrt{\mathcal{L}}}{\sqrt{\sigma_{s}^{0}\left|V_{e N}\right|^{2}+\sigma_{B}}} .
$$

In the above, $\sigma_{s}^{0}$ is the signal cross section for unit mixing, and $\sigma_{B}$ is the background cross section. $\mathcal{L}$ is the required luminosity to achieve $n_{s} \sigma$ significance. Using the above equation, we derive the bound on active-sterile mixing, that we show in Fig. 13. We consider $\mathcal{L}=500 \mathrm{fb}^{-1}$ and $n_{s}=3$. The variable $m_{l j}$ is the invariant mass of the jet and lepton and consequently peaks at a heavy neutrino mass. As shown in Fig. 13, the BDT $5 \sigma(\mathrm{A})$ curve is obtained using the $m_{\ell j}$ variable along with other variables. Being uncorrelated with the standard variables, the use of $m_{\ell j}$ improves the significance, albeit slightly. Similar to the cut-based analysis, we also show the bounds for BDT analysis. Note that the bound from BDT is a factor of 3 stronger than the cut-based analysis. We find that a heavy neutrino of mass 900-1200 GeV and mixing $\left|V_{e N}\right|^{2}=(2.8-5.3) \times 10^{-5}$ can be discovered with $5 \sigma$ significance $\left[\left|V_{e N}\right|^{2}=\right.$ $(1.5-3.0) \times 10^{-5}$ for $\left.3 \sigma\right]$ using $\mathcal{L}=500 \mathrm{fb}^{-1}$ luminosity at $\sqrt{s}=1.4 \mathrm{TeV}$ c.m. energy. A more massive heavy neutrino of mass $M_{N}=1700-2700 \mathrm{GeV}$ and mixing $\left|V_{e N}\right|^{2}=(1.5-3.5) \times 10^{-5}$ can be discovered with $5 \sigma$ significance $\left[\left|V_{e N}\right|^{2}=(0.8-1.1) \times 10^{-5}\right.$ for $\left.3 \sigma\right]$ at $\sqrt{s}=$ $3 \mathrm{TeV}$ c.m. energy using $\mathcal{L}=500 \mathrm{fb}^{-1}$ of data. So far in our analysis we have not used the jet-lepton invariant mass cut. This mass cut enhances the signal significance. As a result, this improves the mass vs mixing bound by 5\%-30\%. This has been shown in Fig. 13. For comparison, we also show the present LHC limits. As can be seen, the leptonic collider is much more effective than the hadronic collider to constrain the mixing angle for higher masses. In [38], the authors analyzed the discovery prospect of a heavy neutrino at the HL-LHC using substructure analysis. For higher masses, the sensitivity reach is $\left|V_{l N}\right|^{2} \sim$ $10^{-1}-10^{-2}$. We find that for heavier $N$, the $e^{+} e^{-}$collider

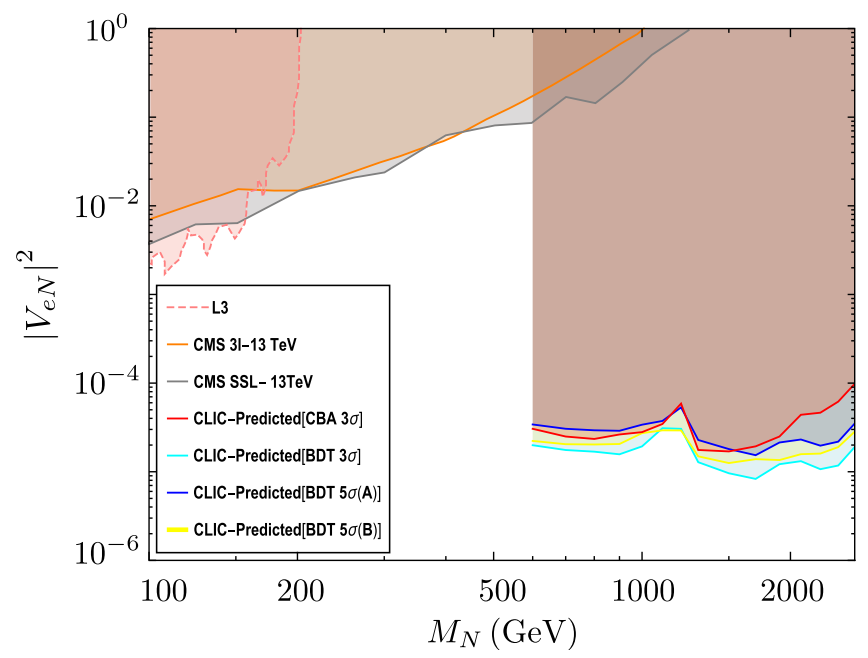

FIG. 13. Limits on the active-sterile mixing vs mass of the heavy neutrino. The different bounds correspond to the CMS $3 \ell+E_{T}$ search [63], CMS $2 \ell+j j[61]$, the limit from LEP [108]. The limits from the cut-based analysis and BDT are in agreement with Tables III, IV, VI, and VII. The CLIC-Predicted

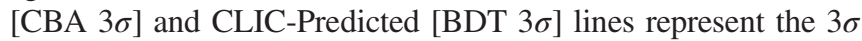
limit, obtained using the cut-based and BDT analysis, respectively. These two limits have been derived without using the jet-lepton invariant mass variable as an input of BDT. CLICPredicted [BDT $5 \sigma(\mathrm{A})]$ represents the $5 \sigma$ sensitivity and it has also been derived without the jet-lepton invariant mass variable. CLIC-Predicted [BDT $5 \sigma(\mathrm{B})]$ corresponds to $5 \sigma$ sensitivity, where, in addition to other variables, the jet-lepton invariant mass has also been used. 
can probe up to a much lower value of active-sterile mixing and hence will have a better sensitivity reach.

\section{CONCLUSION}

We explore the discovery prospect of a heavy neutrino with intermediate and large mass ranges $M_{N}=600-1200$ and $1300-2700 \mathrm{GeV}$ at the proposed $e^{+} e^{-}$collider for two different c.m. energies, $\sqrt{s}=1.4$ and $3 \mathrm{TeV}$, respectively. The heavy neutrino can be produced at the $e^{+} e^{-}$collider through the $s$ - and $t$-channel processes, $e^{+} e^{-} \rightarrow \nu_{e} N$, and decays subsequently. We consider the decay mode with highest branching ratio $N \rightarrow e^{ \pm} W^{\mp}$. The produced $W^{ \pm}$ gauge bosons are highly boosted, and hence their decays produce collimated decay products. We consider the hadronic final states of the produced $W^{ \pm}$'s that lead to fat jets. The model signature is therefore $e^{ \pm}+j_{\text {fat }}+\not p$. For the background, we generate the events as $e^{ \pm} \nu_{e} / \bar{\nu}_{e} j j$, which can come from the $W^{ \pm} W^{\mp}$ sample, but also has other contributions.

For the $\sqrt{s}=1.4 \mathrm{TeV}$ analysis, we use optimized cuts to probe the mass regions $M_{N}=600-900$ and 1000$1200 \mathrm{GeV}$. The charged lepton produced from $N$ has a relatively larger $p_{T}$ for the $1000-1200 \mathrm{GeV}$ mass range. The cuts on the $p_{T}$ of leptons, as well as other variables, such as $\eta^{\ell}, \Delta R(j, \ell)$, and $M_{\mathrm{inv}}^{(p, \ell)}$, remove the majority of the SM background. We find that the entire mass range 600-1100 GeV has a fairly large signal cross section $\sigma_{D}=0.51-0.82 \mathrm{fb}$, after taking into account the detector effect. For the background, the cross section falls after all the cuts, from $751 \mathrm{fb}$ as a partonic cross section to $\sigma_{D} \sim 1 \mathrm{fb}$. In addition to the cut-based analysis, we also pursue multivariate analysis. We find that the heavy neutrino of mass $M_{N}=600-1200 \mathrm{GeV}$ and the activesterile mixing $\left|V_{e N}\right|^{2} \sim 10^{-5}$ can be discovered at $5 \sigma$ significance with $500 \mathrm{fb}^{-1}$ luminosity.
Similar to this analysis, we also pursue the analysis for $\sqrt{s}=3 \mathrm{TeV}$ c.m. energy, using the same set of tools. We explore the mass range $1300-2700 \mathrm{GeV}$ for this case. For this ultraheavy $M_{N}$, the produced $e^{ \pm}$and $W^{ \pm}$'s are even more boosted. The lepton and fat jets have very high $p_{T}$. Typically, for $M_{N}=2100 \mathrm{GeV}$, the peak in $p_{T}$ occurs around $1000 \mathrm{GeV}$. We use cuts on different kinematic variables and follow a MVA prescription. We find that a heavy neutrino of mass $M_{N}=1300-2700 \mathrm{GeV}$ with mixing $\left|V_{e N}\right|^{2} \sim 10^{-5}$ can be discovered at $5 \sigma$ significance with $500 \mathrm{fb}^{-1}$ luminosity.

We stress that the discovery prospect of a heavy neutrino with a $\mathrm{TeV}$ mass is significantly better in a lepton collider, which can operate with higher c.m. energy, as compared to the LHC. This is to note that, in this case, the signal originating from heavy neutrino decay in the semileptonic channel will be very different, as compared to the usual dilepton + dijet signal that has been extensively discussed in the literature. For such a heavy massive neutrino, the produced gauge bosons will be highly boosted, and as a result, the final state will contain a fat-jet signature, as opposed to the resolved jets. To summarize our findings, we find that a heavy neutrino up to mass $2.7 \mathrm{TeV}$ and active-sterile mixing $\left|V_{e N}\right|^{2} \sim 10^{-5}$ can be discovered (with $5 \sigma$ significance) at a lepton collider, operating with 1.4 or $3 \mathrm{TeV}$ c.m. energy, and with $500 \mathrm{fb}^{-1}$ of luminosity. Finally, the studied model signature is relevant, irrespective of the Majorana or pseudo-Dirac nature of heavy neutrinos.

\section{ACKNOWLEDGMENTS}

M. M. acknowledges the support of the DST-INSPIRE Research Grant No. IFA14-PH-99, and the HPC cluster facility at IOP, Bhubaneswar. S. C. is supported by the U.S. Department of Energy under Grant No. de-sc0010102. The authors acknowledge the hospitality of IISER Bhopal during WHEPP-XV, where this work has been initiated.
[1] P. F. De Salas, S. Gariazzo, O. Mena, C. A. Ternes, and M. Trtola, Neutrino mass ordering from oscillations and beyond: 2018 status and future prospects, Front. Astron. Space Sci. 5, 36 (2018).

[2] R. Adam et al. (Planck Collaboration), Planck 2015 results. I. Overview of products and scientific results, Astron. Astrophys. 594, A1 (2016).

[3] S. Weinberg, Baryon and Lepton Nonconserving Processes, Phys. Rev. Lett. 43, 1566 (1979).

[4] F. Wilczek and A. Zee, Operator Analysis of Nucleon Decay, Phys. Rev. Lett. 43, 1571 (1979).

[5] E. Ma, Pathways to Naturally Small Neutrino Masses, Phys. Rev. Lett. 81, 1171 (1998).
[6] F. Bonnet, M. Hirsch, T. Ota, and W. Winter, Systematic study of the $d=5$ Weinberg operator at one-loop order, J. High Energy Phys. 07 (2012) 153.

[7] D. A. Sierra, A. Degee, L. Dorame, and M. Hirsch, Systematic classification of two-loop realizations of the Weinberg operator, J. High Energy Phys. 03 (2015) 040.

[8] A. Zee, A theory of lepton number violation, neutrino Majorana mass, and oscillation, Phys. Lett. 93B, 389 (1980).

[9] R. Barbier et al., R-parity violating supersymmetry, Phys. Rep. 420, 1 (2005).

[10] P. Minkowski, $\mu \rightarrow$ er at a rate of one out of $10^{9}$ muon decays?, Phys. Lett. 67B, 421 (1977). 
[11] R. N. Mohapatra and G. Senjanović, Neutrino Mass and Spontaneous Parity Violation, Phys. Rev. Lett. 44, 912 (1980).

[12] T. Yanagida, Horizontal symmetry and masses of neutrinos, Conf. Proc. C7902131, 95 (1979).

[13] M. Gell-Mann, P. Ramond, and R. Slansky, Complex spinors and unified theories, Conf. Proc. C790927, 315 (1979).

[14] J. Schechter and J. W.F. Valle, Neutrino masses in $\mathrm{SU}(2) \times \mathrm{U}(1)$ theories, Phys. Rev. D 22, 2227 (1980).

[15] K. S. Babu, C. N. Leung, and J. T. Pantaleone, Renormalization of the neutrino mass operator, Phys. Lett. B 319, 191 (1993).

[16] S. Antusch, M. Drees, J. Kersten, M. Lindner, and M. Ratz, Neutrino mass operator renormalization in two Higgs doublet models and the MSSM, Phys. Lett. B 525, 130 (2002).

[17] M. Magg and C. Wetterich, Neutrino mass problem and gauge hierarchy, Phys. Lett. 94B, 61 (1980).

[18] T. P. Cheng and L.-F. Li, Neutrino masses, mixings and oscillations in $\mathrm{SU}(2) \times \mathrm{U}(1)$ models of electroweak interactions, Phys. Rev. D 22, 2860 (1980).

[19] G. Lazarides, Q. Shafi, and C. Wetterich, Proton lifetime and Fermion masses in an SO(10) model, Nucl. Phys. B181, 287 (1981).

[20] R. N. Mohapatra and G. Senjanović, Neutrino masses and mixings in gauge models with spontaneous parity violation, Phys. Rev. D 23, 165 (1981).

[21] R. Foot, H. Lew, X. G. He, and G. C. Joshi, Seesaw neutrino masses induced by a triplet of leptons, Z. Phys. C 44, 441 (1989).

[22] R. N. Mohapatra and J. C. Pati, Left-right gauge symmetry and an isoconjugate model of $C P$ violation, Phys. Rev. D 11, 566 (1975).

[23] R. N. Mohapatra and J. C. Pati, A natural left-right symmetry, Phys. Rev. D 11, 2558 (1975).

[24] G. Senjanovic and R. N. Mohapatra, Exact left-right symmetry and spontaneous violation of parity, Phys. Rev. D 12, 1502 (1975).

[25] R. N. Mohapatra, Mechanism for Understanding Small Neutrino Mass in Superstring Theories, Phys. Rev. Lett. 56, 561 (1986).

[26] R. N. Mohapatra and J. W. F. Valle, Neutrino mass and baryon number nonconservation in superstring models, Phys. Rev. D 34, 1642 (1986).

[27] S. Nandi and U. Sarkar, A Solution to the Neutrino Mass Problem in Superstring E6 Theory, Phys. Rev. Lett. 56, 564 (1986).

[28] F. del Aguila and J. A. Aguilar-Saavedra, Distinguishing seesaw models at LHC with multi-lepton signals, Nucl. Phys. B813, 22 (2009).

[29] D. Fargion, M. Y. Khlopov, R. V. Konoplich, and R. Mignani, On the possibility of searching for heavy neutrinos at accelerators, Phys. Rev. D 54, 4684 (1996).

[30] A. Atre, T. Han, S. Pascoli, and B. Zhang, The search for heavy Majorana neutrinos, J. High Energy Phys. 05 (2009) 030 .

[31] Y. Cai, T. Han, T. Li, and R. Ruiz, Lepton number violation: Seesaw models and their collider tests, Front. Phys. 6, 40 (2018).
[32] A. Datta, M. Guchait, and A. Pilaftsis, Probing lepton number violation via Majorana neutrinos at hadron supercolliders, Phys. Rev. D 50, 3195 (1994).

[33] C. Degrande, O. Mattelaer, R. Ruiz, and J. Turner, Fullyautomated precision predictions for heavy neutrino production mechanisms at hadron colliders, Phys. Rev. D 94, 053002 (2016).

[34] M. Mitra, R. Ruiz, D. J. Scott, and M. Spannowsky, Neutrino jets from high-mass $W_{R}$ gauge bosons in TeVscale left-right symmetric models, Phys. Rev. D 94, 095016 (2016).

[35] S. Pascoli, R. Ruiz, and C. Weiland, Safe jet vetoes, Phys. Lett. B 786, 106 (2018).

[36] P. S. Bhupal Dev and Y. Zhang, Displaced vertex signatures of doubly charged scalars in the type-II seesaw and its left-right extensions, J. High Energy Phys. 10 (2018) 199.

[37] F. F. Deppisch, P. S. Bhupal Dev, and A. Pilaftsis, Neutrinos and collider physics, New J. Phys. 17, 075019 (2015).

[38] A. Bhardwaj, A. Das, P. Konar, and A. Thalapillil, Looking for minimal inverse seesaw scenarios at the LHC with jet substructure techniques, arXiv:1801.00797.

[39] A. Das, P. Konar, and A. Thalapillil, Jet substructure shedding light on heavy Majorana neutrinos at the LHC, J. High Energy Phys. 02 (2018) 083.

[40] A. Abada, N. Bernal, M. Losada, and X. Marcano, Inclusive displaced vertex searches for heavy neutral leptons at the LHC, J. High Energy Phys. 01 (2019) 093.

[41] G. Cottin, J. C. Helo, and M. Hirsch, Displaced vertices as probes of sterile neutrino mixing at the LHC, Phys. Rev. D 98, 035012 (2018).

[42] J. C. Helo, M. Hirsch, and Z. S. Wang, Heavy neutral fermions at the high-luminosity LHC, J. High Energy Phys. 07 (2018) 056.

[43] E. Accomando, L. Delle Rose, S. Moretti, E. Olaiya, and C. H. Shepherd-Themistocleous, Extra Higgs boson and Z? as portals to signatures of heavy neutrinos at the LHC, J. High Energy Phys. 02 (2018) 109.

[44] F. F. Deppisch, W. Liu, and M. Mitra, Long-lived heavy neutrinos from Higgs decays, J. High Energy Phys. 08 (2018) 181.

[45] Z. Kang, P. Ko, and J. Li, New Avenues to heavy right-handed neutrinos with pair production at hadronic colliders, Phys. Rev. D 93, 075037 (2016).

[46] P. S. B. Dev, D. Kim, and R. N. Mohapatra, Disambiguating seesaw models using invariant mass variables at hadron colliders, J. High Energy Phys. 01 (2016) 118.

[47] A. Das, Searching for the minimal seesaw models at the LHC and beyond, Adv. High Energy Phys. 2018, 9785318 (2018).

[48] M. Mitra, G. Senjanovic, and F. Vissani, Neutrinoless double beta decay and heavy sterile neutrinos, Nucl. Phys. B856, 26 (2012).

[49] P. S. Bhupal Dev, S. Goswami, and M. Mitra, TeV scale left-right symmetry and large mixing effects in neutrinoless double beta decay, Phys. Rev. D 91, 113004 (2015).

[50] W. Rodejohann, Neutrinoless double beta decay and neutrino physics, J. Phys. G 39, 124008 (2012).

[51] H. Ps and W. Rodejohann, Neutrinoless double beta decay, New J. Phys. 17, 115010 (2015). 
[52] M. Gonzlez, M. Hirsch, and S. Kovalenko, Neutrinoless double beta decay and QCD running at low energy scales, Phys. Rev. D 97, 115005 (2018).

[53] A. Das, P. S. B. Dev, and R. N. Mohapatra, Same sign versus opposite sign dileptons as a probe of low scale seesaw mechanisms, Phys. Rev. D 97, 015018 (2018).

[54] A. Das, P. Konar, and S. Majhi, Production of heavy neutrino in next-to-leading order QCD at the LHC and beyond, J. High Energy Phys. 06 (2016) 019.

[55] A. Abada, C. Biggio, F. Bonnet, M. B. Gavela, and T. Hambye, Low energy effects of neutrino masses, J. High Energy Phys. 12 (2007) 061.

[56] A. Abada, C. Biggio, F. Bonnet, M. B. Gavela, and T. Hambye, mu $\rightarrow$ e gamma and tau $\rightarrow 1$ gamma decays in the fermion triplet seesaw model, Phys. Rev. D 78, 033007 (2008).

[57] A. Ali, A. V. Borisov, and N. B. Zamorin, Majorana neutrinos and same sign dilepton production at LHC and in rare meson decays, Eur. Phys. J. C 21, 123 (2001).

[58] S. Mandal and N. Sinha, Favoured $B_{c}$ decay modes to search for a Majorana neutrino, Phys. Rev. D 94, 033001 (2016).

[59] S. Mandal, M. Mitra, and N. Sinha, Constraining the righthanded gauge boson mass from lepton number violating meson decays in a low scale left-right model, Phys. Rev. D 96, 035023 (2017).

[60] W.-Y. Keung and G. Senjanovic, Majorana Neutrinos and the Production of the Right-Handed Charged Gauge Boson, Phys. Rev. Lett. 50, 1427 (1983).

[61] A. M. Sirunyan et al. (CMS Collaboration), Search for heavy Majorana neutrinos in same-sign dilepton channels in proton-proton collisions at $\sqrt{s}=13 \mathrm{TeV}$, J. High Energy Phys. 01 (2019) 122.

[62] F. del Aguila and J. A. Aguilar-Saavedra, Electroweak scale seesaw and heavy Dirac neutrino signals at LHC, Phys. Lett. B 672, 158 (2009).

[63] A. M. Sirunyan et al. (CMS Collaboration), Search for Heavy Neutral Leptons in Events with Three Charged Leptons in Proton-Proton Collisions at $\sqrt{s}=13 \mathrm{TeV}$, Phys. Rev. Lett. 120, 221801 (2018).

[64] S. Mondal and S. K. Rai, Probing the heavy neutrinos of inverse seesaw model at the LHeC, Phys. Rev. D 94, 033008 (2016).

[65] S. Mondal and S. K. Rai, Polarized window for left-right symmetry and a right-handed neutrino at the large hadronelectron collider, Phys. Rev. D 93, 011702 (2016).

[66] M. Lindner, F. S. Queiroz, W. Rodejohann, and C. E. Yaguna, Left-right symmetry and lepton number violation at the large hadron electron collider, J. High Energy Phys. 06 (2016) 140.

[67] F. del Aguila and J. A. Aguilar-Saavedra, $1 \mathrm{~W}$ nu production at CLIC: A window to TeV scale non-decoupled neutrinos, J. High Energy Phys. 05 (2005) 026.

[68] F. del Aguila, J. A. Aguilar-Saavedra, A. Martinez de la Ossa, and D. Meloni, Flavor and polarisation in heavy neutrino production at $e^{+} e^{-}$colliders, Phys. Lett. B 613, 170 (2005).

[69] A. Das and N. Okada, Inverse seesaw neutrino signatures at the LHC and ILC, Phys. Rev. D 88, 113001 (2013).
[70] S. Banerjee, P. S. B. Dev, A. Ibarra, T. Mandal, and M. Mitra, Prospects of heavy neutrino searches at future lepton colliders, Phys. Rev. D 92, 075002 (2015).

[71] S. Antusch, E. Cazzato, M. Drewes, O. Fischer, B. Garbrecht, D. Gueter, and J. Klarić, Probing leptogenesis at future colliders, J. High Energy Phys. 09 (2018) 124.

[72] S. Antusch, E. Cazzato, and O. Fischer, Sterile neutrino searches at future $e^{-} e^{+}, p p$, and $e^{-} p$ colliders, Int. J. Mod. Phys. A 32, 1750078 (2017).

[73] S. Antusch, E. Cazzato, and O. Fischer, Displaced vertex searches for sterile neutrinos at future lepton colliders, J. High Energy Phys. 12 (2016) 007.

[74] S. Antusch and O. Fischer, Testing sterile neutrino extensions of the Standard Model at future lepton colliders, J. High Energy Phys. 05 (2015) 053.

[75] S. Antusch, E. Cazzato, and O. Fischer, Higgs production from sterile neutrinos at future lepton colliders, J. High Energy Phys. 04 (2016) 189.

[76] P. Hernndez, J. Jones-Prez, and O. Surez-Navarro, Majorana vs pseudo-Dirac neutrinos at the ILC, Eur. Phys. J. C 79, 220 (2019).

[77] S. S. Biswal and P. S. B. Dev, Probing left-right seesaw models using beam polarization at an $e^{+} e^{-}$collider, Phys. Rev. D 95, 115031 (2017).

[78] E. Accomando et al. (CLIC Physics Working Group Collaboration), Physics at the CLIC multi-TeV linear collider, in Proceedings, 11th International Conference on Hadron spectroscopy (Hadron 2005): Rio de Janeiro, Brazil, August 21-26, 2005 (2004), http://weblib.cern.ch/ abstract?CERN-2004-005.

[79] L. Linssen, A. Miyamoto, M. Stanitzki, and H. Weerts, Physics and detectors at CLIC: CLIC conceptual design report, arXiv:1202.5940.

[80] H. Abramowicz et al. (CLIC Detector and Physics Study Collaboration), Physics at the CLIC $e^{+} e^{-}$linear colliderinput to the Snowmass process 2013, in Proceedings, 2013 Community Summer Study on the Future of U.S. Particle Physics: Snowmass on the Mississippi (CSS2013): Minneapolis, MN, USA, 2013 (2013), http://inspirehep .net/record/1243603/files/arXiv:1307.5288.pdf.

[81] N. A. Tehrani, J.-J. Blaising, B. Cure, D. Dannheim, F. D. Ramos, K. Elsener et al., CLICdet: The post-CDR CLIC detector model, CLICdp-Note-2017-001, 2017.

[82] R. Contino, C. Grojean, D. Pappadopulo, R. Rattazzi, and A. Thamm, Strong Higgs interactions at a linear collider, J. High Energy Phys. 02 (2014) 006.

[83] S. Heinemeyer and C. Schappacher, Neutral Higgs boson production at $e^{+} e^{-}$colliders in the complex MSSM: A full one-loop analysis, Eur. Phys. J. C 76, 220 (2016).

[84] A. Thamm, R. Torre, and A. Wulzer, Future tests of Higgs compositeness: Direct vs indirect, J. High Energy Phys. 07 (2015) 100.

[85] N. Craig, M. Farina, M. McCullough, and M. Perelstein, Precision Higgsstrahlung as a probe of new physics, J. High Energy Phys. 03 (2015) 146.

[86] G. Durieux, C. Grojean, J. Gu, and K. Wang, The leptonic future of the Higgs, J. High Energy Phys. 09 (2017) 014.

[87] J. Ellis, P. Roloff, V. Sanz, and T. You, Dimension-6 operator analysis of the CLIC sensitivity to new physics, J. High Energy Phys. 05 (2017) 096. 
[88] D. Dannheim, P. Lebrun, L. Linssen, D. Schulte, F. Simon, S. Stapnes et al., CLIC $e^{+} e^{-}$linear collider studies, arXiv: 1208.1402.

[89] M. Thomson, Model-independent measurement of the $e^{+}$ $e^{-} \rightarrow \mathrm{HZ}$ cross section at a future $e^{+} e^{-}$linear collider using hadronic Z decays, Eur. Phys. J. C 76, 72 (2016).

[90] G. Milutinović-Dumbelović, I. Božović-Jelisavčić, C. Grefe, G. Kačarević, S. Lukić, M. Pandurović, P. Roloff, and I. Smiljanić, Physics potential for the measurement of $\sigma(H \nu \bar{\nu}) \times \operatorname{BR}\left(H \rightarrow \mu^{+} \mu^{-}\right)$at the $1.4 \mathrm{TeV}$ CLIC collider, Eur. Phys. J. C 75, 515 (2015).

[91] H. Wang and B. Yang, Top partner production at $e^{+} e^{-}$ collider in the littlest Higgs model with T-parity, Adv. High Energy Phys. 2017, 5463128 (2017).

[92] H. Abramowicz et al., Higgs physics at the CLIC electronpositron linear collider, Eur. Phys. J. C 77, 475 (2017).

[93] S. Banerjee, B. Bhattacherjee, M. Mitra, and M. Spannowsky, The lepton flavour violating Higgs decays at the HL-LHC and the ILC, J. High Energy Phys. 07 (2016) 059.

[94] M. Agostini et al. (GERDA Collaboration), Improved Limit on Neutrinoless Double- $\beta$ Decay of ${ }^{76} \mathrm{Ge}$ from GERDA Phase II, Phys. Rev. Lett. 120, 132503 (2018).

[95] J. Kotila and F. Iachello, Phase space factors for double- $\beta$ decay, Phys. Rev. C 85, 034316 (2012).

[96] A. Meroni, S. T. Petcov, and F. Simkovic, Multiple $C P$ non-conserving mechanisms of $(\beta \beta)_{0 \nu}$-decay and nuclei with largely different nuclear matrix elements, J. High Energy Phys. 02 (2013) 025.

[97] N. D. Christensen and C. Duhr, FeynRules_Feynman rules made easy, Comput. Phys. Commun. 180, 1614 (2009).

[98] A. Alloul, N. D. Christensen, C. Degrande, C. Duhr, and B. Fuks, FeynRules 2.0-A complete toolbox for tree-level phenomenology, Comput. Phys. Commun. 185, 2250 (2014).
[99] C. Degrande, C. Duhr, B. Fuks, D. Grellscheid, O. Mattelaer, and T. Reiter, UFO-The Universal FeynRules output, Comput. Phys. Commun. 183, 1201 (2012).

[100] J. Alwall, R. Frederix, S. Frixione, V. Hirschi, F. Maltoni, O. Mattelaer, H.-S. Shao, T. Stelzer, P. Torrielli, and M. Zaro, The automated computation of tree-level and nextto-leading order differential cross sections, and their matching to parton shower simulations, J. High Energy Phys. 07 (2014) 079.

[101] T. Sjostrand, L. Lonnblad, and S. Mrenna, PYTHIA 6.2: Physics and manual, arXiv:hep-ph/0108264.

[102] J. de Favereau, C. Delaere, P. Demin, A. Giammanco, V. Lemaítre, A. Mertens, and M. Selvaggi (DELPHES 3 Collaboration), DELPHES 3, A modular framework for fast simulation of a generic collider experiment, J. High Energy Phys. 02 (2014) 057.

[103] Y. L. Dokshitzer, G. D. Leder, S. Moretti, and B. R. Webber, Better jet clustering algorithms, J. High Energy Phys. 08 (1997) 001.

[104] A. Bogert and M. Battagila, Jet reconstruction at highenergy electronpositron colliders, https://indico.cern.ch/ event/107327/contributions/30333/attachments/23057/ 33240/09-27presfab.pdf.

[105] M. Boronat, J. Fuster, I. Garcia, P. Roloff, R. Simoniello, and M. Vos, Jet reconstruction at high-energy electronpositron colliders, Eur. Phys. J. C 78, 144 (2018).

[106] M. A. Weber, Jet performance at CLIC, http://cds.cern.ch/ record/2648827.

[107] J. S. Marshall, A. Münnich, and M. A. Thomson, Performance of particle flow calorimetry at CLIC, Nucl. Instrum. Methods Phys. Res., Sect. A 700, 153 (2013).

[108] P. Achard et al. (L3 Collaboration), Search for heavy isosinglet neutrino in $e^{+} e^{-}$annihilation at LEP, Phys. Lett. B 517, 67 (2001). 